\title{
Mixing between ordinary and exotic fermions
}

\author{
Paul Langacker* and David London \\ Deutsches Elektronen Synchrotron-DESY, Hamburg, Federal Republic of Germany
}

(Received 18 April 1988)

\begin{abstract}
The results of a comprehensive analysis of the limits on mixings between ordinary fermions and possible heavy fermions with exotic $S U(2) \times U(1)$ assignments (e.g., left-handed singlets and/or right-handed doublets) are presented. A general formalism for describing such mixings is given. It is shown that a variety of constraints, including the relation between the $W$ and $Z$ masses and the Fermi constant, charged-current universality, limits on induced right-handed charged currents, and flavor-diagonal neutral currents suffice to limit all directions in parameter space that are not excluded by the absence of flavor-changing neutral currents. Limits on $s^{2}$, the square of the mixing between ordinary and exotic fermions, are quite stringent for the $v_{\mu L}, \mu_{L}^{-}, u_{L}$, and $d_{L}$ $\left(s^{2} \leq 0.002-0.005\right)$ if only one particle is allowed to mix at a time, but are weaker by an order of magnitude if fine-tuned cancellations between different mixings are allowed. Similar statements apply to quark mixings with heavy sequential doublets. Limits on $s^{2}$ for the other light fermions $\left(v_{e L}\right.$, $\left.e_{\bar{L}}^{-}, e_{\bar{R}}^{-}, \mu_{R}^{-}, u_{R}, d_{R}\right)$ are in the range $0.02-0.06$, while those for the $s, c, b, v_{\tau}$, and $\tau^{-}$are considerably weaker. Slightly stronger limits are found in specific models (e.g., $\mathrm{E}_{6}$ ). Implications for the masses of the heavy exotic fermions are discussed.
\end{abstract}

\section{INTRODUCTION}

Many of the possibilities for new physics beyond the standard model predict the existence of new fermions, often with exotic $\mathrm{SU}(2) \times \mathbf{U}(1)$ assignments. In addition to direct production, these new fermions can manifest themselves through their mixing with the ordinary (known) quarks and leptons. In this paper we present a systematic analysis of the limits on such mixings that are implied by the many high-precision experiments involving charged-current and flavor-diagonal neutral-current processes, as well as the $W$ and $Z$ masses. (Constraints from limits on flavor-changing neutral currents, which are generally more stringent but can be evaded by finetuning, are also presented.) It will be seen that the limits on mixings are fairly stringent, at least for $v_{e}, e^{-}, v_{\mu}, \mu^{-}$, $u$, and $d$, but that the corresponding constraints on the masses of exotic new fermions (implied for reasonable relations between the masses and mixings) are rather weak. There have been a number of earlier analyses of limits on mixings ${ }^{1}$ (all completed, however, before the last generation of high-precision neutral-current experiments ${ }^{2}$ ), but these have all been restricted to specific models or to specific types of fermions, such as neutrinos, leptons, or quarks only. The present analysis is very general, applying to all models that do not involve exotic electric charge or color assignments, and allowing all of the known fermions to mix with exotic partners simultaneously. Surprisingly, the limits obtained in the general case are almost as stringent as those for specific models (such as those restricted to exotic fermions occurring in 27-plets of $\mathrm{E}_{6}$ or mirror fermions with a Hermitian mass matrix), which are also presented for comparison.

In the standard model, ${ }^{3}$ the three families of known left-handed $(L)$ fermions are assigned to doublet representations of weak SU(2),

$$
\begin{aligned}
& \left(\begin{array}{l}
v_{e} \\
e^{-}
\end{array}\right)_{L},\left(\begin{array}{l}
v_{\mu} \\
\mu^{-}
\end{array}\right)_{L},\left(\begin{array}{l}
v_{\tau} \\
\tau^{-}
\end{array}\right)_{L}, \\
& {\left[\begin{array}{l}
u \\
d
\end{array}\right]_{L},\left(\begin{array}{l}
c \\
s
\end{array}\right)_{L},\left(\begin{array}{l}
t \\
b
\end{array}\right)_{L},}
\end{aligned}
$$

while the right-handed $(R)$ fermions are $\mathrm{SU}(2)$ singlets:

$$
\begin{array}{llllll}
e_{R}^{-}, & \mu_{R}^{-}, & \tau_{R}^{-}, & c_{R}, & c_{R}, & t_{R}, \\
d_{R}, & s_{R}, & b_{R}
\end{array}
$$

where we have assumed the existence of the $t$ and $v_{\tau}$. In (1) and (2) and the rest of Sec. I, pairs of particles enclosed in parentheses refer to SU(2) doublets, while those not enclosed are singlets.

There are several possibilities for new fermions, as shown in Table I. Sequential fermions are simply repetitions of the known fermions, with canonical ( $L$-doublet, $R$-singlet) $\mathrm{SU}(2) \times \mathrm{U}(1)$ assignments. One usually considers complete sequential families (quarks plus leptons). Sequential fourth-family fermions have received much recent phenomenological and theoretical attention. ${ }^{4}$

There are several classes of possible fermions with noncanonical $\mathrm{SU}(2) \times \mathbf{U}(1)$ assignments. For example, mirror fermions $^{5}$ have the opposite $S U(2)$ assignments from those of normal fermions, i.e., $L$-handed singlets and $R$ handed doublets. Usually one considers full mirror families (quarks plus leptons). Mirror fermions occur naturally in some grand unified theories [such as SO(18) models $^{6}$ ], and appear necessary for a consistent formulation of the (chiral) standard model on the lattice ${ }^{7}$ (though it is not clear whether they should be interpreted as real or as a lattice artifact). Most often, however, they are introduced ad hoc in models to cancel anomalies.

Vector doublets refer to new quarks or leptons for which both the $L$ - and $R$-handed components are SU(2) 
TABLE I. Possible types of new fermions. Pairs of particles enclosed in parentheses are SU(2) doublets, while other particles (not enclosed) are SU(2) singlets. $N$ and $E^{-}$refer to any new color singlets (leptons) with electric charges 0 and -1 , respectively, while $U$ and $D$ represent new color triplets (quarks) with electric charges $\frac{2}{3}$ and $-\frac{1}{3}$.

$$
\begin{aligned}
& \text { Sequential fermions } \\
& \left(\begin{array}{c}
N \\
E^{-}
\end{array}\right]_{L} E_{R}^{-},\left(\begin{array}{l}
U \\
D
\end{array}\right)_{L} \begin{array}{l}
U_{R} \\
D_{R}
\end{array}
\end{aligned}
$$

Noncanonical $\mathrm{SU}(2) \times \mathrm{U}(1)$ assignments

(a) Mirror fermions

$$
\begin{aligned}
& E_{L}^{-}\left(\begin{array}{c}
N \\
E^{-}
\end{array}\right)_{R}, \quad D_{L} \quad\left(\begin{array}{ll}
U \\
D
\end{array}\right)_{R} \\
& \text { (b) Vector doublets } \\
& \left(\begin{array}{c}
N \\
E^{-}
\end{array}\right)_{L}\left[\begin{array}{c}
N \\
E^{-}
\end{array}\right)_{R},\left(\begin{array}{l}
U \\
D
\end{array}\right)_{L}\left(\begin{array}{l}
U \\
D
\end{array}\right)_{R} \\
& \text { (c) Vector singlets } \\
& \begin{array}{llllll}
E_{L}^{-} & E_{R}^{-}, & U_{L} & U_{R}, & D_{L} & D_{R}
\end{array} \\
& \text { (d) Weyl Neutrinos } \\
& N_{L}, N_{R} \\
& \text { Exotic electric charges } \\
& {\left[\begin{array}{l}
Q^{5 / 3} \\
U^{2 / 3}
\end{array}\right]_{L},\left[\begin{array}{l}
D^{-1 / 3} \\
Q^{-4 / 3}
\end{array}\right]_{L}, \quad\left[\begin{array}{c}
E^{-} \\
L^{--}
\end{array}\right]_{L}, \quad \cdots}
\end{aligned}
$$

doublets. Similarly, vector singlets are new particles $\left(E^{-}, U\right.$, or $D$ ) for which both $L$ and $R$ are singlets.

Finally, one can introduce new SU(2)-singlet Weyl neutrinos $N_{L}$ (or $N_{R}$ ). These, as well as new doublet neutrinos (sequential, mirror, or vector doublet), can be massless or heavy. In the latter case one can have Majorana masses or one can combine pairs of Weyl neutrinos to form massive Dirac neutrinos.

TABLE II. Fermion content of an $\mathrm{E}_{6}$ 27-plet of left-handed fields. Shown also are the $\mathrm{SO}(10)$ and $\mathrm{SU}(5)$ representations. $v_{e L}^{c}, N_{L}$, and $s_{L}$ are new Weyl neutrinos, $E^{-}$is a charged lepton, and $D$ has the same charge and color as the $d$ quark. The superscript $c$ refers to antiparticle. The right-handed particles are related to the left-handed antiparticles by $\psi_{R}=C \bar{\psi}_{L}^{c}{ }^{T}$, where $C$ is the charge-conjugation matrix.

\begin{tabular}{ccc}
\hline \hline $\mathrm{SO}(10)$ & $\mathrm{SU}(5)$ & $\mathrm{SU}(2) \times \mathrm{U}(1)$ \\
\hline 16 & $5^{*}$ & $\left(\begin{array}{c}v_{e} \\
e^{-}\end{array}\right)_{L} d_{L}^{c}$ \\
& 10 & $u_{L}^{c}\left(\begin{array}{c}u \\
d\end{array}\right)_{L} e_{L}^{+}$ \\
10 & 1 & $\left.\begin{array}{c}v_{e L}^{c} \\
E^{+} \\
N^{c}\end{array}\right]_{L} D_{L}$ \\
1 & 5 & $D_{L}^{c}\left(\begin{array}{c}N \\
E^{-}\end{array}\right)_{L}$ \\
& $5^{*}$ & $s_{L}$ \\
\hline \hline
\end{tabular}

As an example, consider an $\mathrm{E}_{6}$ model with each fermion family assigned to a 27-plet, as shown in Table II. Each 27-plet contains, in addition to the 15 ordinary fermions, (i) a new vector singlet $D$ quark: $D_{L}, D_{R}$, (ii) a new vector doublet of leptons

$$
\left(\begin{array}{c}
N \\
E^{-}
\end{array}\right)_{L}, \quad\left[\begin{array}{c}
N \\
E^{-}
\end{array}\right)_{R},
$$

and (iii) two new Weyl neutrinos $v_{e L}^{c}, s_{L}$.

Finally, one can have models with exotic electric charges (as illustrated in Table I) or noncanonical color assignments. We will not consider such models in this paper.

The limits on new fermions from direct production at accelerators are fairly weak. One has ${ }^{8}$ roughly

$$
M_{N}>18 \mathrm{GeV}, M_{E^{-}}, M_{U}, M_{D}>23 \mathrm{GeV}
$$

from DESY PETRA and SLAC PEP, though in some cases the limits may be weaker depending on the (modeldependent) lifetimes and decay modes. It should be possible to set lower bounds $\sim 40 \mathrm{GeV}$ for some of the models in Table I using recent UA1 (Ref. 9) and UA2 (Ref. 10) direct production data, but again the limits would depend on the details of the decays and have not been systematically analyzed. Similar comments apply to indirect determinations of the ratio $\Gamma_{Z} / \Gamma_{W}$ (Ref. 11). Future colliders should extend the sensitivity of direct searches substantially. ${ }^{12}$

Mixing between ordinary fermions and heavy fermions with noncanonical $S U(2) \times U(1)$ assignments will usually induce flavor-changing neutral currents (FCNC's) between the ordinary fermions. In particular, the existing limits on $d \leftrightarrow s, c \leftrightarrow u, b \leftrightarrow d, b \leftrightarrow s$, and $\mu \leftrightarrow e$ FCNC's are extremely stringent. We will summarize these limits in Sec. II, but we have little to add over previous analyses. However, there are certain (fine-tuned) directions in parameter space (those in which each ordinary mass eigenstate mixes with a distinct linear combination of heavy fermions) in which no FCNC's are induced. Our major concern in this paper is to show that these remaining directions can all be limited (rather stringently for the $v_{e}$, $e^{-}, v_{\mu}, \mu^{-}, u$, and $d$ ) by existing charged-current and flavor-diagonal neutral-current data. In particular, charged-current universality, limits on induced righthanded currents, and the relation between $M_{W}, M_{Z}$, and the Fermi constant put strong constraints on the possible mixings of the lighter left-handed fermions with exotic $L$ singlets, while neutral-current data limit the possible mixings of the right-handed and heavy $\left(c_{L}, b_{L}, \tau_{L}\right)$ lefthanded particles.

It is easy to see that in the absence of exotic electric charge and color assignments the sequential fermions and noncanonical SU $(2) \times \mathbf{U}(1)$ models in Table $I$ are the most general possibilities for new fermions. ${ }^{13}$ In particular, the only possible exotic $S U(2) \times U(1)$ assignments are lefthanded singlets, which can occur in mirror or vector singlet models, or right-handed doublets, which occur in mirror and vector doublet models. (The neutrinos are slightly more complicated because of the possibility of Majorana masses.) In this paper we will mainly be con- 
cerned with the mixing between these and the ordinary ( $L$-doublet, $R$-singlet) fermions of (1). In Sec. II we will develop a formalism that is sufficiently powerful to deal with all of these cases. In general, the mixing between $n$ ordinary and $m$ exotic fermions must be described by a unitary $(n+m) \times(n+m)$ matrix. However, we will show that the observed absence of FCNC between the ordinary quarks and charged leptons implies an enormous simplification: all of the remaining (non-FCNC) mixings can be parametrized by one effective mixing angle for each ordinary (left- or right-handed) particle.

Things are more complicated for the neutrinos. This is partly because there is no empirical evidence to justify the neglect of FCNC's between the ordinary neutrinos. Fortunately, such an assumption is not needed: we will show that because one must sum over the unobserved flavors of the final neutrinos in weak processes, one can still describe most mixing effects by one effective mixing angle per ordinary neutrino. The other complication is that there are more possibilities for the $S U(2) \times U(1)$ representations of exotic neutrinos, i.e., in the general Majorana case, the left-handed neutrinos $N_{1 L}, N_{2 L}, N_{3 L}$ in the representations

$$
\left(\begin{array}{l}
N_{1} \\
E^{-}
\end{array}\right)_{L}, \quad\left[\begin{array}{l}
E^{+} \\
N_{2}
\end{array}\right)_{L}, N_{3 L}
$$

can all mix (Dirac neutrinos are a limiting case). This complicates the analysis of neutral-current data because $N_{1}$ and $N_{2}$ have nonzero but different neutral-current couplings. We show that this effect can be parametrized by one (bounded) auxiliary parameter per neutrino. Throughout we will assume that all neutrinos (ordinary and exotic) are either "massless" (i.e., with masses too small to be kinematically relevant to present experiments) or "heavy" (i.e., too heavy to be produced in normal weak decays or to be important in present scattering experiments). In particular, we assume that the $v_{\mu}$ and $v_{\tau}$ are massless or very light. This is actually the most conservative assumption for deriving upper limits on mixings. If it should turn out that $m_{v_{\mu}}$ and/or $m_{v_{\tau}}$ are actually "large" (i.e., near their present limits ${ }^{14}$ or $\sim 250 \mathrm{keV}$ and $\sim 35 \mathrm{MeV}$, respectively), then the upper limits on mixings with exotic heavy neutrinos would become stronger, because the kinematic effects of neutrino mass are similar to those of mixing (and of the same sign) for most of the experiments considered here.

In Sec. III we present the charged-and neutral-current data that we use. It will be seen that there are a large number of rather stringent constraints, but that care is needed in the analysis to ensure that all effects of mixings [e.g., on the determination of subsidiary parameters, such as ordinary (Cabibbo-type) quark mixing angles or flux normalizations] are properly included.

In Sec. IV we describe the results of the fits. We show that there are enough constraints to simultaneously determine the weak mixing angle $\sin ^{2} \theta_{W}$ and to limit the mixings of all of the ordinary $L$ - and $R$-handed fermions. We present the results of several types of fits, such as those in which only one particle is allowed to mix at a time, those in which all of the particles can mix with exotic partners simultaneously (allowing for fine-tuned cancellations between the effects of different mixings), and those in which additional model-dependent constraints are imposed. We also discuss the implications of the mixing limits for the masses of the heavy exotic particles, using typical expected relations between masses and mixings.

In Sec. $\mathrm{V}$ we summarize the results, and the Appendix is devoted to some technical details concerning the formalism.

A related topic is that the mixing between ordinary and exotic neutrinos can induce total lepton-number violation and individual lepton-flavor violation in the ordinary neutrino sector, even if the ordinary $v$ 's are massless. ${ }^{15,16}$ The phenomenological implications will be discussed separately. ${ }^{16}$

\section{FORMALISM}

Let us first treat the quarks and charged leptons (neutrinos are considered separately below). The most general $\mathrm{SU}(2) \times \mathbf{U}(1)$ assignments not involving exotic charges or colors are shown in Table I. We will classify all fermions as either ordinary or exotic according to their $\mathbf{S U}(2)$ transformation properties. In particular, we define all left-handed particles occurring in doublets to be "ordinary" (independent of whether the doublets are associated with known or sequential families, or with vector doublets), and all left-handed SU(2) singlets (mirror families or vector singlets) to be "exotic." Similarly, we define right-handed singlets (known, sequential, or vector singlet) to be "ordinary" and right-handed doublets (mirror or vector doublets) to be "exotic."

It is convenient to arrange all of the left-handed charge $-\frac{2}{3}$ quark fields in the theory into a column vector

$$
u_{L}^{0} \equiv\left(u_{1 L}^{0} \quad u_{2 L}^{0} \cdots u_{(n+m) L}^{0}\right)^{T},
$$

and similarly for $u_{R}^{0}, d_{L}^{0}, d_{R}^{0}, e_{L}^{-0}$, and $e_{R}^{-0}$. In (5) we assume there are $n$ ordinary and $m$ exotic $u_{L}^{0}$ fields. The superscript zero refers to the weak-interaction basis-i.e., the original fields in the Lagrangian with definite $\mathbf{S U}(2) \times \mathbf{U}(1)$ transformation properties. Rather than treating $u^{0}, d^{0}$, and $e^{-0}$ separately or adding an extra index, we will introduce the generic vectors $\psi_{L}^{0}$ and $\psi_{R}^{0}$, which can represent either $u_{L, R}^{0}, d_{L, R}^{0}$, or $e_{L, R}^{-0} \cdot \psi_{L, R}^{0}$ can be decomposed into ordinary and exotic sectors by

$$
\psi_{L}^{0}=\left(\begin{array}{l}
\psi_{O L}^{0} \\
\psi_{E L}^{0}
\end{array}\right), \quad \psi_{R}^{0}=\left(\begin{array}{l}
\psi_{O R}^{0} \\
\psi_{E R}^{0}
\end{array}\right)
$$

In (6), $\psi_{O L}^{0}$ is a column vector consisting of $n_{L}$ ordinary [SU(2)-doublet] fields, while $\psi_{E L}^{0}$ consists of $m_{L}$ exotic [SU(2)-singlet] fields. Similarly, $\psi_{O R}^{0}$ consists of $n_{R}$ ordinary [SU(2)-singlet] states, and $\psi_{E R}^{0}$ consists of $m_{R}$ exotic [SU(2)-doublet] states. $n$ and $m$ may be different for $u, d$, and $e$, but one must of course have $n_{L}^{u}=n_{L}^{d}$ and $m_{R}^{u}=m_{R}^{d}$. The additional physical requirement that one 
have massive Dirac quarks and charged leptons requires $n_{L}^{i}+m_{L}^{i}=n_{R}^{i}+m_{R}^{i}, i=u, d, e$.

In each sector there will also be $n_{L}^{i}+m_{L}^{i}$ masseigenstate fields, which can be arranged in column vectors

$$
\psi_{L} \equiv\left(\begin{array}{lll}
\psi_{1 L} & \cdots & \psi_{(n+m) L}
\end{array}\right)^{T}
$$

and

$$
\psi_{R} \equiv\left(\begin{array}{lll}
\psi_{1 R} & \cdots & \psi_{(n+m) R}
\end{array}\right)^{T} .
$$

We assume that $\psi_{L}$ (and $\psi_{R}$ ) can be decomposed as

$$
\psi_{L}=\left(\begin{array}{l}
\psi_{l L} \\
\psi_{h L}
\end{array}\right), \quad \psi_{R}=\left(\begin{array}{c}
\psi_{l R} \\
\psi_{h R}
\end{array}\right),
$$

where $\psi_{l L}$ is a vector of $n_{L}$ "light"-mass eigenstates, $\psi_{h L}$ represents $m_{L}$ "heavy"-mass eigenstates, and similarly for $\psi_{l R}, \psi_{h R}$. The interpretation of this ansatz is that we expect that there will be $n$ light states composed mainly of the $n$ ordinary weak eigenstates $\psi_{o}^{0}$, and $m$ heavy states consisting mainly of the $m$ exotic states $\psi_{E}^{0}$, with relatively little mixing between the two sectors. In fact, since we have included such things as sequential and vector doublets into $\psi_{O L}^{0}$ (and similarly for $\psi_{O R}^{0}$ ), it is not clear that the mass eigenstates will always cleanly separate into two sectors of very different masses. The labels "light" and "heavy" should, therefore, be considered suggestive only. The decomposition in (7) will be defined more precisely below [see Eq. (18)].

The weak and mass eigenstates are related by

$$
\psi_{L}^{0}=U_{L} \psi_{L}, \quad \psi_{R}^{0}=U_{R} \psi_{R},
$$

where $U_{L}$ and $U_{R}$ are the $(n+m) \times(n+m)$-dimensional unitary matrices $\left(U_{a}^{\dagger} U_{a}=U_{a} U_{a}^{\dagger}=I, a=L, R\right)$ which diagonalize the fermion mass matrix. (When necessary, we will add a superscript to distinguish the unitary transformations for $u, d$, and $e^{-}$.) One can write $U_{L}$ and $U_{R}$ in block form as

$$
U_{a}=\left(\begin{array}{ll}
A_{a} & E_{a} \\
F_{a} & G_{a}
\end{array}\right), \quad a=L, R,
$$

so that

$$
\begin{aligned}
& {\left[\begin{array}{l}
\psi_{O L}^{0} \\
\psi_{E L}^{0}
\end{array}\right)=\left(\begin{array}{ll}
A_{L} & E_{L} \\
F_{L} & G_{L}
\end{array}\right]\left[\begin{array}{l}
\psi_{l L} \\
\psi_{h L}
\end{array}\right],} \\
& \left(\begin{array}{l}
\psi_{O R}^{0} \\
\psi_{E R}^{0}
\end{array}\right]=\left[\begin{array}{ll}
A_{R} & E_{R} \\
F_{R} & G_{R}
\end{array}\right]\left[\begin{array}{l}
\psi_{l R} \\
\psi_{h R}
\end{array}\right] .
\end{aligned}
$$

In (9) and (10), $A_{a}$ is an $\left(n_{a} \times n_{a}\right)$-dimensional matrix relating the ordinary weak states and the light-mass eigenstates, while $G_{a}$ is an $\left(m_{a} \times m_{a}\right)$-dimensional matrix relating the exotic and heavy states. $E_{a}$ and $F_{a}$, which are $\left(n_{a} \times m_{a}\right)$ - and $\left(m_{a} \times n_{a}\right)$-dimensional, respectively, describe the mixing between the two sectors. We will refer to $E_{a}$ and $F_{a}$ as the "light-heavy" mixing matrices. The matrices $A_{a}$ and $G_{a}$ are not unitary. Rather, from the unitarity of $U_{a}$, one has

$$
\begin{aligned}
& A_{a}^{\dagger} A_{a}+F_{a}^{\dagger} F_{a}=I, \\
& A_{a} A_{a}^{\dagger}+E_{a} E_{a}^{\dagger}=I, \quad a=L, R,
\end{aligned}
$$

with similar relations for $G_{a}$. We anticipate that all of the components of $F_{a}$ and $E_{a}$ will be small, so that the deviation of $A_{a}$ from unitarity is of second order in light-heavy mixing. Many of the physical effects of mixing with exotic fermions are associated with the nonunitarity of $A_{a}$.

The part of the weak neutral current involving $u^{0}, d^{0}$, or $e^{-0}$ is

$$
\begin{aligned}
\frac{1}{2} J_{Z}^{\mu}= & \bar{\psi}_{L}^{0} \gamma^{\mu}\left(P_{Z}^{L} t_{3}-\sin ^{2} \theta_{W} I q\right) \psi_{L}^{0} \\
& +\bar{\psi}_{R}^{0} \gamma^{\mu}\left(P_{Z}^{R} t_{3}-\sin ^{2} \theta_{W} I q\right) \psi_{R}^{0} \\
= & \bar{\psi}_{O L}^{0} \gamma^{\mu} t_{3} \psi_{O L}^{0}+\bar{\psi}_{E R}^{0} \gamma^{\mu} t_{3} \psi_{E R}^{0}-\sin ^{2} \theta_{W} J_{\mathrm{em}}^{\mu},
\end{aligned}
$$

where

$$
P_{Z}^{L}=\left(\begin{array}{ll}
I & 0 \\
0 & 0
\end{array}\right), \quad P_{Z}^{R}=\left(\begin{array}{ll}
0 & 0 \\
0 & I
\end{array}\right)
$$

are projection operators onto the subspaces of ordinary $L$ fields and exotic $R$ fields, respectively,

$$
t_{3}= \begin{cases}+\frac{1}{2}, & u, \\ -\frac{1}{2}, & d, e^{-}\end{cases}
$$

is the $T_{3}$ eigenvalue of the $\psi_{O L}^{0}$ and $\psi_{E R}^{0}, q=\frac{2}{3}(u)$, $-\frac{1}{3}(d),-1(e)$ is the charge, and $J_{\mathrm{em}}^{\mu}$ is the (flavordiagonal) electromagnetic current. Since we are considering mixing between doublets and singlets (i.e., $J_{Z}^{\mu}$ involves $P_{Z}^{L, R}$ rather than the identity), there is no GlashowIliopoulos-Maiani (GIM) mechanism, ${ }^{17}$ and there will be FCNC's, in general. Rewriting $J_{Z}^{\mu}$ in terms of mass eigenstates using (10), one obtains

$$
\begin{aligned}
\frac{1}{2} J_{Z}^{\mu}= & \bar{\psi}_{l L} \gamma^{\mu} t_{3} A_{L}^{\dagger} A_{L} \psi_{l L}+\bar{\psi}_{l R} \gamma^{\mu} t_{3} F_{R}^{\dagger} F_{R} \psi_{l R} \\
& +\bar{\psi}_{l L} \gamma^{\mu} t_{3} A_{L}^{\dagger} E_{L} \psi_{h L}+\bar{\psi}_{l R} \gamma^{\mu} t_{3} F_{R}^{\dagger} G_{R} \psi_{h R} \\
& +\bar{\psi}_{h L} \gamma^{\mu} t_{3} E_{L}^{\dagger} A_{L} \psi_{l L}+\bar{\psi}_{h R} \gamma^{\mu} t_{3} G_{R}^{\dagger} F_{R} \psi_{l R} \\
& +\bar{\psi}_{h L} \gamma^{\mu} t_{3} E_{L}^{\dagger} E_{L} \psi_{h L}+\bar{\psi}_{h R} \gamma^{\mu} t_{3} G_{R}^{\dagger} G_{R} \psi_{h R} \\
& -\sin ^{2} \theta_{W} J_{\mathrm{em}}^{\mu} .
\end{aligned}
$$

The last $\left(J_{\mathrm{em}}^{\mu}\right)$ term is flavor diagonal, since all fields which mix have the same electric charge. The second and third lines represent flavor-changing neutral-current transitions between light- and heavy-mass eigenstates. Such transitions are phenomenologically harmless, ${ }^{18}$ although they would be of great importance for the production and decay of the heavy fermions.

Much more dangerous are the first two terms in $J_{Z}^{\mu}$. The first is the normal $t_{3}$ part of the neutral current, modified by $A_{L}^{\dagger} A_{L} \neq I$. In addition to changing the strength of the diagonal terms $\left(A_{L}^{\dagger} A_{L}\right)_{i i}=1-\left(F_{L}^{\dagger} F_{L}\right)_{i i}$ $\leq 1$, one generally expects FCNC transitions $\bar{\psi}_{i L} \gamma^{\mu} \psi_{j L} Z_{\mu}$ between the light fermions $\psi_{i}$ and $\psi_{j}$, with strength 


$$
\lambda_{i j}^{L} \equiv\left(A_{L}^{\dagger} A_{L}\right)_{i j}=-\left(F_{L}^{\dagger} F_{L}\right)_{i j}, \quad i \neq j
$$

i.e., of second order in light-heavy mixing. Similarly, the second term in $J_{Z}^{\mu}$ is an induced right-handed component of the neutral current, with off-diagonal (and diagonal) transitions of strength

$$
\lambda_{i j}^{R} \equiv\left(F_{R}^{\dagger} F_{R}\right)_{i j},
$$

which is again second order in light-heavy mixing. It is possible that $\lambda_{i j}^{L, R}$ vanish for $i \neq j$, but there is no $a$ priori reason to expect them to do so.

Limits ${ }^{19-22}$ on $\lambda_{i j}^{L, R}$ are extremely stringent for $s \dot{d}, c u$, $b d, b s$, and $\mu e$, and non-negligible for $\tau \mu$ and $\tau e$, as can be seen in Table III. We will, therefore, take it as a physical assumption that there are no FCNC's involving the light fermions, i.e.,

$$
\lambda_{i j}^{L}=\lambda_{i j}^{R}=0 \text { for } i \neq j
$$

This ansatz is, of course, not directly tested for $\lambda_{t u}, \lambda_{t c}$, or transitions involving sequential fermions, $L$-vector doublets, or $\boldsymbol{R}$-vector singlets. However, it seems to us unlikely that deviations from (18) in such cases will cause much trouble. For example, limits on the mixing between $d_{L}$ and a heavy vector singlet are unlikely to be greatly affected by the presence of a small FCNC between $d_{L}$ and a heavy fourth family $D_{L}$.

The absence of FCNC's assumed in (18) allows an enormous simplification. In particular, the matrices $A_{L}^{\dagger} A_{L}$ and $F_{R}^{\dagger} F_{R}$ are diagonal, and from the unitarity relations (11) it is easy to show that $0 \leq\left(A_{L}^{\dagger} A_{L}\right)_{i i} \leq 1$ and $0 \leq\left(F_{R}^{\dagger} F_{R}\right)_{i i} \leq 1$. Hence, one can write

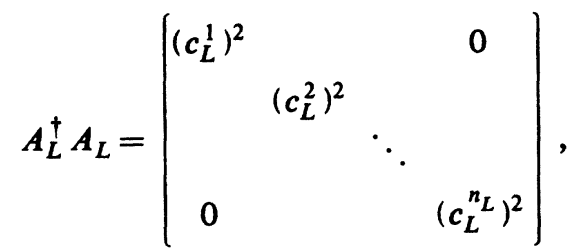

$$
\begin{aligned}
& F_{R}^{\dagger} F_{R}=\left(\begin{array}{cccc}
\left(s_{R}^{1}\right)^{2} & & & 0 \\
& \left(s_{R}^{2}\right)^{2} & & \\
& & \ddots & \\
0 & & & \left(s_{R}^{n_{R}}\right)^{2}
\end{array}\right) \text {, }
\end{aligned}
$$

where $c_{L}^{i} \equiv \cos \theta_{L}^{i}$ and $s_{R}^{i} \equiv \sin \theta_{R}^{i}$, where $\theta_{L}^{i}$ and $\theta_{R}^{i}$ will be interpreted as light-heavy mixing angles.

It is convenient to define the diagonal matrices

$$
\begin{aligned}
& c_{a}=\operatorname{diag}\left(\begin{array}{llll}
c_{a}^{1} & c_{a}^{2} & \cdots & c_{a}^{n_{a}}
\end{array}\right), \\
& s_{a}=\operatorname{diag}\left(\begin{array}{llll}
s_{a}^{1} & s_{a}^{2} & \cdots & s_{a}^{n_{a}}
\end{array}\right), \quad a=L, R,
\end{aligned}
$$

so that [using (11)] $A_{L}^{\dagger} A_{L}=c_{L}^{2}, A_{R}^{\dagger} A_{R}=c_{R}^{2}, F_{L}^{\dagger} F_{L}=s_{L}^{2}$, and $F_{R}^{\dagger} F_{R}=s_{R}^{2}$.

A rough interpretation of (19) is that there are no FCNC's if and only if the light-heavy mixing is restricted
TABLE III. Limits on flavor-changing neutral-current parameters $\lambda_{i j}$ defined in (16) and (17). We have used the limits on the coefficients of effective operators (derived for an entirely different purpose) given in Ref. 19, as well as new experimental results for leptonic transitions and $B \rightarrow l^{+} l^{-} X$ reviewed in Refs. 20-22. The limit on $\lambda_{b s}$ is from searches for $B \rightarrow l^{+} l^{-} X$. There are no constraints from $B_{s}-\bar{B}_{s}$ mixing, which is expected to be nearly maximal in the standard model.

\begin{tabular}{lll}
\hline \hline Quantity & Upper limit & \multicolumn{1}{c}{ Source } \\
\hline$\left|\lambda_{d s}\right|$ & $3 \times 10^{-4}$ & $\Delta m_{K_{L} K_{S}}$ \\
& $6 \times 10^{-5}$ & $K_{L} \rightarrow \mu^{+} \mu^{-}$ \\
$\left|\lambda_{c u}\right|$ & $5 \times 10^{-4}$ & $D^{0}-\bar{D}^{0}$ \\
$\left|\lambda_{b d}\right|$ & $4 \times 10^{-4}$ & $B_{d}-\bar{B}_{d}$ \\
$\left|\lambda_{\mu e}\right|$ & $3 \times 10^{-6}$ & $\mu \nrightarrow 3 e$ \\
$\left|\lambda_{\tau \mu}\right|,\left|\lambda_{\tau e}\right|$ & $4 \times 10^{-2}$ & $\tau \rightarrow 3 l$ \\
$\left|\lambda_{b s}\right|$ & $1 \times 10^{-2}$ & $B \nrightarrow l+l^{-} X$ \\
\hline \hline
\end{tabular}

to distinct pairs of states, i.e., each light state mixes with its own heavy state (which need not be a mass eigenstate), with mixing angle $\theta_{L}^{i}$ or $\theta_{R}^{i}$. This is made more precise in the following three theorems and in the Appendix.

Theorem 1. $A_{a}, a=L, R$, can be written as

$$
A_{a}=\widehat{A}_{a} c_{a}=\widehat{A}_{a}\left(\begin{array}{ccc}
c_{a}^{1} & & 0 \\
& \ddots & \\
0 & & c_{a}^{n_{a}}
\end{array}\right) \text {, }
$$

where $\hat{A}_{a}$ is unitary $\left(\hat{A}_{a}^{\dagger} \hat{A}_{a}=\hat{A}_{a} \hat{A}_{a}^{\dagger}=I\right)$.

That is, assuming the absence of FCNC's, $A_{a}$ can be factorized into a unitary matrix $\hat{A}_{a}$, which describes normal intergenerational mixing, and a diagonal matrix of $\cos \theta_{a}^{i}$, where $\theta_{a}^{i}$ is the light-heavy mixing angle for $\psi_{i a}$. The proof is given in the Appendix.

Theorem 2. $F_{a}, a=L, R$, can be written as

$$
F_{a}=\hat{F}_{a} s_{a}=\hat{F}_{a}\left(\begin{array}{ccc}
s_{a}^{1} & & 0 \\
& \ddots & \\
0 & & s_{a}^{n_{a}}
\end{array}\right) .
$$

$\hat{F}_{a}$ is unitary in the special case $n_{a}=m_{a}$.

Again, there is a factorization into an intergenerational term and a diagonal matrix of light-heavy mixing angles. The proof and a discussion of the $m_{a} \neq n_{a}$ case are given in the Appendix.

Theorem 3. One can choose a weak-eigenstate basis such that $\hat{A}_{a}=I$ and (for $\left.n_{a}=m_{a}\right) \hat{F}_{a}=I$.

That is, one can always pick the original weak eigenstates so that each corresponds to a unique light-mass eigenstate. In the following we will always choose $\hat{A}_{a}^{e}=I, \hat{F}_{a}^{e}=I$. One can also choose the quark weak basis so that, for example, $\hat{A}_{L}^{u}=I$ or $\hat{A}_{L}^{d}=I$ but, in general, not both simultaneously. The proof and a discussion of the $n_{a} \neq m_{a}$ case are given in the Appendix.

From (15) and (19), the neutral current for the light quarks and charged leptons is 


$$
\begin{aligned}
\frac{1}{2} J_{Z}^{\mu}=\sum_{i}[ & \bar{\psi}_{i L} \gamma^{\mu}\left(t_{3} c_{L}^{i^{2}}-\sin ^{2} \theta_{W} q\right) \psi_{i L} \\
& \left.+\bar{\psi}_{i R} \gamma^{\mu}\left(t_{3} s_{R}^{i^{2}}-\sin ^{2} \theta_{W} q\right) \psi_{i R}\right],
\end{aligned}
$$

where we have dropped the subscript $l$. The $c_{L}^{i^{2}}$ term represents a nonuniversal reduction of the strength of the normal neutral current, due to mixing with $L$ singlets, and the $s_{R}^{i^{2}}$ is an induced right-handed current. The latter is more important than the analogous induced right-handed charged current because it can interfere with the existing right-handed current (in $J_{\mathrm{em}}^{\mu}$ ) in deepinelastic processes.

Similarly, the part of the hadronic weak charged current involving light-mass eigenstates is

$$
\frac{1}{2} J_{W}^{\mu^{\dagger}}=\bar{u}_{L} \gamma^{\mu} V_{L} d_{L}+\bar{u}_{R} \gamma^{\mu} V_{R} d_{R},
$$

where $u_{L}$ and $d_{L}$ are $n$-component column vectors of the light-mass eigenstate left-handed quarks of charge $\frac{2}{3}$ and $-\frac{1}{3}$, respectively, and similarly for $u_{R}, d_{R}$. In (24)

$$
V_{L}=A_{L}^{u^{\dagger}} A_{L}^{d}
$$

is the apparent Kobayashi-Maskawa-Cabibbo (KMC) quark mixing matrix, ${ }^{23}$ which is nonunitary in the presence of mixing with exotic fermions. Using (21),

$$
V_{L}=c_{L}^{u} \hat{A}_{L}^{u_{L}^{\dagger}} \hat{A}_{L}^{d} c_{L}^{d}=c_{L}^{u} \widehat{V}_{L} c_{L}^{d},
$$

where $\widehat{V}_{L}=\widehat{A}_{L}^{u^{\dagger}} \widehat{A}_{L}^{d}$ is the true (unitary) KMC mixing matrix. We always use the term true to refer to underlying quantities (in this case, the unitary intergeneration mixing matrix), and apparent to refer to the directly measured quantities. The two, which are represented by symbols with and without a caret, respectively, differ by light-heavy mixing effects. From (26), the effects of light-heavy mixing are represented by a nonuniversal reduction in strength (i.e., $V_{L_{i j}}=c_{L}^{u_{i}} c_{L}^{d_{j}} \widehat{V}_{L_{i j}}$ ), while the effects of extra sequential families (or quark-vector doublets) are manifested by an apparent nonunitarity in $\hat{V}_{L}$ when the effects of the extra doublets are not taken into consideration.

The second term in $J_{W}^{\mu}$ is the induced right-handed current, described by the matrix

$$
V_{R}=F_{R}^{u^{\dagger}} F_{R}^{d}=s_{R}^{u} \hat{F}_{R}^{u^{\dagger}} \hat{F}_{R}^{d} s_{R}^{d} \equiv s_{R}^{u} \hat{V}_{R} s_{R}^{d} .
$$

$V_{R}$ is of second order in light-heavy mixing, since both the $u_{i}$ and $d_{j}$ must mix into the $R$ doublet. The righthanded term is important for processes such as $\beta$ and $K_{l 3}$ decay, since it has a vector component. However, it is unimportant for deep-inelastic scattering because there are no standard model right-handed currents to interfere with (i.e., it only contributes to order $s_{R}^{4}$ in cross sections). The matrix $\hat{V}_{R}=\hat{F}_{R}^{u^{\dagger}} \hat{F}_{R}^{d}$ is unitary ${ }^{24}$ for $n_{R}^{u}=n_{R}^{d}=m_{R}^{u}$.

The neutrinos must be treated separately because of the possibility of Majorana masses and because there are no experimental constraints on possible FCNC's.

In dealing with Majorana neutrinos it is convenient to denote all left-handed neutrinos by $n_{L}$ and all righthanded neutrinos by $n_{R}^{c}$. The two are not independent but are related by

$$
n_{R}^{c}=C\left(\bar{n}_{L}\right)^{T},
$$

where $C$ is the charge-conjugation matrix defined by $C \gamma_{\mu} C^{-1}=-\gamma_{\mu}^{T}$. Under a $C P$ transformation, $n_{L}(\mathrm{x}, t)$ $\rightarrow \gamma^{0} n_{R}^{c}(-\mathrm{x}, t)$, so that $n_{L}$ and $n_{R}^{c}$ are essentially $C P$ conjugates. For the usual neutrinos, $n_{L}$ and $n_{R}^{c}$ are what are commonly referred to as left-handed neutrinos and righthanded antineutrinos, respectively. More generally, however, the superscript $c$ simply means that $n_{L}$ and $n_{R}^{c}$ are $C P$ conjugates. It does not imply that the $n_{L}$ are all leptons and the $n_{R}^{c}$ are antileptons. There is no real distinction between them for Majorana neutrinos, and for the special case of Dirac neutrinos $n_{L}$ refers to both lefthanded neutrinos and left-handed antineutrinos. ${ }^{25}$

Assuming that there are no exotic electric charges, there are three possibilities for the weak-eigenstate $L$ neutrinos, which we denote by $n_{O L}^{0}, n_{E L}^{0}$, and $n_{S L}^{0}$. The $n_{O L}^{0}$ are the ordinary $L$ doublets

$$
\left[\begin{array}{l}
n_{O L}^{0} \\
e_{L}^{0^{-}}
\end{array}\right) \leftrightarrow\left(\begin{array}{l}
e_{R}^{0^{+}} \\
n_{O R}^{0^{c}}
\end{array}\right)
$$

related by $C P$ to $R$ doublets involving positrons. There are two kinds of exotic neutrinos. The $n_{E L}^{0}$ are related by $C P$ to exotic $R$ doublets:

$$
\left(\begin{array}{l}
e_{L}^{0^{+}} \\
n_{E L}^{0}
\end{array}\right) \leftrightarrow\left(\begin{array}{c}
n_{E R}^{0^{c}} \\
e_{R}^{0^{-}}
\end{array}\right),
$$

while

$$
n_{S L}^{0} \leftrightarrow n_{S R}^{0^{c}}
$$

represent any $\mathrm{SU}(2)$ singlets. In the general Majorana case all three kinds can mix. In the Dirac case there is no mixing between $n_{O L}^{0}$ and $n_{E L}^{0}$, and the $n_{S L}^{0}$ divide into two sectors with lepton numbers +1 and -1 ; these can mix with $n_{O L}^{0}$ and $n_{E L}^{0}$, respectively.

In analogy to (6) we arrange all of the weak-eigenstate neutrinos into a vector

$$
n_{L}^{0}=\left(\begin{array}{l}
n_{O L}^{0} \\
n_{E L}^{0} \\
n_{S L}^{0}
\end{array}\right), \quad n_{R}^{0^{c}}=\left(\begin{array}{c}
n_{O R}^{0^{c}} \\
n_{E R}^{0^{c}} \\
n_{S R}^{0^{c}}
\end{array}\right),
$$

where $n_{O L}^{0}, \quad n_{E L}^{0}$, and $n_{S L}^{0}$ are $n^{v}, m^{v}$, and $p^{v_{-}}$ dimensional vectors, respectively. Of course, one must have $n^{v}=n_{L}^{e}$ and $m^{v}=m_{R}^{e}$.

We assume that the mass-eigenstate neutrinos are all either "massless" (i.e., with masses too small to be kinematically relevant) or heavy. As argued in Sec. I, this is a conservative assumption for deriving upper limits on mixings. We write

$$
n_{L}=\left[\begin{array}{l}
n_{l L} \\
n_{h L}
\end{array}\right], \quad n_{R}^{c}=\left[\begin{array}{l}
n_{l R}^{c} \\
n_{h R}^{c}
\end{array}\right],
$$

where the $n_{l L}$ are $q$ "massless" neutrinos and the $n_{h L}$ are $r$ heavy neutrinos. In (33), the mass eigenstates are as- 
sumed to be Majorana two-component neutrinos. Dirac neutrinos are obtained by combining pairs of degenerate Majorana $v$ 's (Ref. 14).

The two bases are related by a unitary transformation

$$
n_{L}^{0}=U_{L} n_{L}, \quad n_{R}^{0^{c}}=U_{R} n_{R}^{c},
$$

where $^{14,26} U_{L}=U_{R}^{*}$. $U_{L}$ can be written in block form

$$
U_{L}=\left(\begin{array}{ll}
A_{L} & E_{L} \\
F_{L} & G_{L} \\
H_{L} & J_{L}
\end{array}\right),
$$

where $A_{L}, F_{L}$, and $H_{L}$ are $(n \times q)-,(m \times q)-$, and $(p \times q)$-dimensional matrices describing the overlap of the massless $v$ 's with ordinary $\left(n_{O L}^{0}\right)$ doublets, exotic $\left(n_{E L}^{0}\right)$ doublets, and singlets $\left(n_{S L}^{0}\right)$, respectively. Similarly, $E_{L}$, $G_{L}$, and $J_{L}$ are $(n \times r),(m \times r)$, and $(p \times r)$ dimensional. Unlike the quarks and charged leptons we do not necessarily assume that $n=q$. One could imagine the existence of sequential families or vector doublets involving heavy $v$ 's $(n>q)$, for example. Similarly, there could be singlets or mirror families with massless neutrinos $(n<q)$. In the former case some of the components of $A_{L}$ would describe mixing between massless and heavy states (though they are both "ordinary"), while in the latter case some of the components of $F_{L}$ or $H_{L}$ could be large. In practice, however, we will usually be concerned with two special cases. (a) The light neutrinos are all mainly ordinary $n_{O L}^{0}$ states, so that $F_{L}$ and $H_{L}$ are small light-heavy mixings. (b) The light neutrinos are Dirac, consisting of ordinary $n_{O L}^{0}$ states and their SU(2)-singlet partners (i.e., what would normally be called the righthanded neutrino and left-handed antineutrino). The light singlets, which decouple in the absence of mass and mixing effects, correspond to large components of $H_{L}$, while $F_{L}$ and any remaining $H_{L}$ components are light-heavy mixings.

The unitarity of $U_{L}$ implies

$$
A_{L}^{\dagger} A_{L}+F_{L}^{\dagger} F_{L}+H_{L}^{\dagger} H_{L}=I, \quad A_{L} A_{L}^{\dagger}+E_{L} E_{L}^{\dagger}=I
$$

There is no evidence to justify assuming that $A_{L}^{\dagger} A_{L}$ is diagonal. However, one can accomplish much the same purpose by summing over the flavors of unobserved final neutrinos in weak processes.

The weak neutral current for the charged leptons (repeated for convenience) and the light neutrinos is ${ }^{27}$

$$
\begin{aligned}
\frac{1}{2} J_{Z}^{\mu}= & \bar{e}_{L} \gamma^{\mu}\left[-\frac{1}{2}\left(c_{L}^{e}\right)^{2}+\sin ^{2} \theta_{W}\right] e_{L} \\
& +\bar{e}_{R} \gamma^{\mu}\left[-\frac{1}{2}\left(s_{R}^{e}\right)^{2}+\sin ^{2} \theta_{W}\right] e_{R} \\
& +\frac{1}{2} \bar{n}_{L} \gamma^{\mu}\left(A_{L}^{v^{\dagger}} A_{L}^{v}-F_{L}^{v^{\dagger}} F_{L}^{v}\right) n_{L},
\end{aligned}
$$

where $e_{L, R}$ are column vectors of the mass-eigenstate charged leptons, and we have dropped the subscript $l$. The $A_{L}^{\dagger} A_{L}$ and $F_{L}^{\dagger} F_{L}$ terms are the neutral currents of $n_{O L}^{0}$ and $n_{E L}^{0}$, respectively.

The leptonic charged current is ${ }^{27}$

$$
\begin{aligned}
\frac{1}{2} J_{W}^{\mu_{W}^{\dagger}} & =\bar{n}_{L} \gamma^{\mu} A_{L}^{\nu^{\dagger}} c_{L}^{e} e_{L}+\bar{n}_{R}^{c} \gamma^{\mu} F_{R}^{\nu^{\dagger}} s_{R}^{e} e_{R} \\
& =\sum_{i a}\left[\bar{n}_{i L} \gamma^{\mu}\left(A_{L}^{\nu^{\dagger}}\right)_{i a} c_{L}^{e_{a}} e_{a L}+\bar{n}_{i R}^{c} \gamma^{\mu}\left(F_{R}^{\nu^{\dagger}}\right)_{i a} s_{R}^{e_{a}} e_{a R}\right]
\end{aligned}
$$

In (38), $F_{R}^{v}=F_{K}^{v *}$, and we have chosen the weak basis for $e_{L, R}^{-}$so that $\hat{A}_{L}^{e}=I$ and $\hat{F}_{R}^{e}=I$ (Ref. 28). In the lefthanded part of $J_{W}^{\mu^{\dagger}}$ the $e_{a L}^{-}-n_{i L}$ coupling is reduced in strength by the factors $\cos \theta_{L}^{e_{a}}$ and $\left(A_{L}^{v^{\dagger}}\right)_{i a}$ which differ from unity due to electron and neutrino light-heavy mixing, respectively.

The second term in $J_{W}^{\mu^{\dagger}}$ is the induced right-handed current, which can be rewritten

$$
\bar{n}_{R}^{c} \gamma^{\mu} F_{R}^{v^{\dagger}} s_{R}^{e} e_{R}=-\bar{e}_{L}^{c} \gamma^{\mu} s_{R}^{e} F_{L}^{v} n_{L},
$$

where $e_{L}^{c}=C \bar{e}_{R}^{T}$ is the vector of antileptons $\left(e_{L}^{+}, \mu_{L}^{+}, \tau_{L}^{+}\right.$, etc). We will usually (i.e., except for one model considered in Sec. IV) assume that the light neutrinos are mainly the ordinary $n_{O L}^{0}$ states (or $n_{S L}^{0}$ singlets for Dirac neutrinos), so that $F_{L}^{v}$ are small light-heavy mixings. Then the current in (39) is of second order in light-heavy mixing.

Let us write $\Gamma\left(e_{a} \rightarrow n_{i}\right)$ to represent any weak decay involving the $e_{a} \rightarrow n_{i}$ transition (e.g., $K^{+} \rightarrow e_{a}^{c} n_{i}$ ). From (38), the decay rate is changed relative to the normal rate $\Gamma_{0}$ by a factor 29

$$
\begin{aligned}
\frac{1}{\Gamma_{0}} \sum_{i} \Gamma\left(e_{a} \rightarrow n_{i}\right) & =\left(c_{L}^{e_{a}}\right)^{2}\left(A_{L}^{v} A_{L}^{v^{\dagger}}\right)_{a a}+\left(s_{R}^{e_{a}}\right)^{2}\left(F_{R}^{v} F_{R}^{v^{\dagger}}\right)_{a a} \\
& =\left(c_{L}^{e_{a}}\right)^{2}\left(c_{L}^{v_{a}}\right)^{2}+\left(s_{R}^{e_{a}}\right)^{2}\left(s_{R}^{v_{a}}\right)^{2},
\end{aligned}
$$

where we have summed over the flavor of the final neutrino. (There may be additional mixing corrections associated with the other particles in the decay.) In (40) we have introduced the effective neutrino mixing angles

$$
\begin{aligned}
& \left(c_{L}^{v_{a}}\right)^{2}=\cos ^{2} \theta_{L}^{v_{a}} \equiv\left(A_{L}^{v} A_{L}^{\nu^{\dagger}}\right)_{a a}=\sum_{i}\left|\left(A_{L}^{v^{\dagger}}\right)_{i a}\right|^{2}, \\
& \left(s_{R}^{v_{a}}\right)^{2}=\sin ^{2} \theta_{R}^{v_{a}} \equiv\left(F_{R}^{v} F_{R}^{v^{\dagger}}\right)_{a a}=\sum_{i}\left|\left(F_{R}^{v^{\dagger}}\right)_{i a}\right|^{2} .
\end{aligned}
$$

$\left[\left(c_{L}^{v_{a}}\right)^{2}\right.$ and $\left(s_{R}^{v_{a}}\right)^{2}$ are restricted to the range $0-1$ by the unitarity of $U_{L}$.] The first term in (40) is the usual lefthanded current, reduced in strength by $e$ and $v$ mixing. The second is the induced right-handed current (RHC).

We will henceforth work to second order in light-heavy mixing in rates and cross sections (with a few exceptions involving muon, $\tau$, and $\beta$ decay). We can, therefore, drop the $O\left(s^{4}\right)$ RHC term in (40). It is also important that neutrinos are produced with the normal helicity, i.e., the RHC term in (40), which produces the opposite helicity from normal, is negligibly small. (Furthermore, in many cases the cross sections for the subsequent rescattering of the wrong helicity neutrinos are also suppressed by lightheavy mixing. ${ }^{30}$ )

The normalized state produced in $(40)$ is

$$
\left|n_{a L}\right\rangle \equiv \frac{\sum_{i}\left(A_{L}^{v^{\dagger}}\right)_{i a}\left|n_{i L}\right\rangle}{c_{L}^{v_{a}}},
$$


i.e., a coherent superposition of the states $\left|n_{i L}\right\rangle$. Since the $n_{i L}$ are degenerate (massless) this state does not change in time except for an irrelevant overall phase. ${ }^{31}$

We can now consider the cross section for the state $n_{a L}$ produced in (40) to subsequently rescatter in a target. From (38) and (42) the cross section for charged-current scattering into the "right" lepton $e_{a L}$ is

$$
\frac{1}{\sigma_{0}} \sigma\left(n_{a L} \rightarrow e_{a}\right)=\left(c_{L}^{e_{a}}\right)^{2}\left(c_{L}^{v_{a}}\right)^{2},
$$

where $\sigma_{0}$ is the normal cross section in the absence of mixing. Again, the interpretation is that the weakinteraction strength is reduced by mixing of the $e$ and $v$ with noninteracting exotic leptons. In (43) and the other cross sections below there may be additional mixing corrections associated with the target particles.

An interesting consequence of the mixing between light and heavy neutrinos is that the effective states $\left|n_{a L}\right\rangle$ and $\left|n_{b L}\right\rangle$ associated with $e_{a \bar{L}}$ and $e_{b \bar{L}}$ in weak decays need not be orthogonal. ${ }^{15}$ (This is manifested in the nonunitarity of $A_{L}^{v}$.) This leads to lepton-flavor and total leptonnumber violation even if the light neutrinos are exactly massless. For example, the state $n_{a L}$ can rescatter to produce the wrong charged lepton $e_{b}$, with relative cross section

$$
\frac{1}{\sigma_{0}} \sigma\left(n_{a L} \rightarrow e_{b}\right)=\frac{\left(c_{L}^{e_{b}}\right)^{2}\left|\left(A_{L}^{v} A_{L}^{v^{\dagger}}\right)_{b a}\right|^{2}}{\left(c_{L}^{v_{a}}\right)^{2}} .
$$

Reaction (44) would mimic the effects of neutrino oscillations in many appearance experiments, ${ }^{14}$ even though no oscillations actually occur ${ }^{15}$ (i.e., the rate would be independent of energy and distance). The phenomenological implications of lepton-number violation induced by nonorthogonal neutrinos for neutrino-appearance experiments, $\mu \rightarrow e \gamma$, neutrinoless double-beta decay, and muon decay will be discussed elsewhere. ${ }^{16}$

From (37) and (42) the cross section for neutral-current rescattering of the $n_{a L}$, summed over the unobserved final neutrino flavor, is

$$
\frac{1}{\sigma_{0}} \sum_{i} \sigma\left(n_{a L} \rightarrow n_{i L}\right)=\frac{\left[A_{L}^{v}\left(A_{L}^{v^{\dagger}} A_{L}^{v}-F_{L}^{\nu^{\dagger}} F_{L}^{v}\right)^{2} A_{L}^{v^{\dagger}}\right]_{a a}}{\left(c_{L}^{v_{a}}\right)^{2}} .
$$

(Target corrections must also be included.) From (36) and (45) it is clear that the cross section depends not only on the mixing angle, but on whether the mixing is with exotic doublets $\left(F_{L}^{v} A_{L}^{\nu \dagger} \neq 0\right.$; this is a $\Delta L= \pm 2$ mixing that occurs for Majorana neutrinos only), with exotic singlets $\left(H_{L} A_{L}^{\dagger} \neq 0\right.$; this can occur for both Dirac and Majorana neutrinos), with heavy sequential neutrinos (or vector doublets), or with all of these.

Fortunately, to second order in light-heavy mixing, (45) simplifies greatly. It is shown in the Appendix that

$$
\frac{1}{\sigma_{0}} \sum_{i} \sigma\left(n_{a L} \rightarrow n_{i L}\right) \rightarrow 1-\Lambda_{a}\left(s_{L}^{v_{a}}\right)^{2}+O\left(s^{4}\right),
$$

where $\Lambda_{a}$ is an auxiliary parameter that can vary between
0 and 4, depending on the particles involved in the mixing. In the special case of mixing with only one type of heavy neutrino one has

$\Lambda_{a}= \begin{cases}0 & \text { heavy sequential (or vector doublet) in } n_{O L}^{0}, \\ 2 & \text { singlet in } n_{S L}^{0}, \\ 4 & \text { exotic doublet }(\Delta L= \pm 2) \text { in } n_{E L}^{0} .\end{cases}$

As expected, the neutral current is unaffected by mixing with a heavy sequential or vector doublet neutrino.

In the next section we will incorporate the effects of mixing on the target particles and on subsidiary parameters needed to interpret cross-section measurements.

\section{EXPERIMENTAL CONSTRAINTS}

In this section we describe the experimental constraints on mixing. True (underlying) quantities are denoted with a caret, while apparent (measured) quantities, which differ by mixing effects, are uncareted. We work to second order in light-heavy mixing except where otherwise stated. Theoretical expressions are given in Table IV and experimental numbers in Table V.

$$
\text { A. } M_{W}, M_{Z}
$$

The $W$ and $Z$ masses provide an absolute prediction of the strength of the weak interactions in the absence of exotic mixings. One has ${ }^{2}$

$$
M_{W}=\frac{\hat{A}_{0}}{\sin \theta_{W}(1-\Delta r)^{1 / 2}}, \quad M_{Z}=\frac{M_{W}}{\cos \theta_{W}},
$$

where $\sin ^{2} \theta_{W} \equiv 1-M_{W}^{2} / M_{Z}^{2}$ is the renormalized weak angle (using the Sirlin definition $\left.{ }^{32}\right), \hat{A}_{0}=\left(\pi \alpha / \sqrt{2} \hat{G}_{\mu}\right)^{1 / 2}$, and $\hat{G}_{\mu}$ is the (true) Fermi constant. It differs from the value $G_{\mu}=1.16637 \times 10^{-5} \mathrm{GeV}^{-2}$ measured in muon decay by

$$
G_{\mu}=\hat{G}_{\mu} c_{L}^{e} c_{L}^{v_{e}} c_{L}^{\mu} c_{L}^{v_{\mu}}
$$

because the effective four-fermion interaction is reduced in strength by the factors $c_{L}^{i}$ [induced right-handed currents only affect the relation between $G_{\mu}$ and $\hat{G}_{\mu}$ to $O\left(s^{4}\right)$ ]. Hence, $\hat{A}_{0}$ is reduced from its canonical value of $A_{0}=\left(\pi \alpha / \sqrt{2} G_{\mu}\right)^{1 / 2}=37.281 \mathrm{GeV}$ to

$$
\widehat{A}_{0}=A_{0}\left(c_{L}^{e} c_{L}^{v{ }^{e}} c_{L}^{\mu} c_{L}^{v_{\mu}}\right)^{1 / 2}
$$

In (48) $\Delta r$ is a radiative correction parameter predict$\mathrm{ed}^{2}$ to be $0.0713 \pm 0.0013$ for $m_{t}=45 \mathrm{GeV}$ and $m_{H}=100$ $\mathrm{GeV}\left(m_{t}\right.$ and $m_{H}$ are the $t$-quark and Higgs-boson masses, respectively), while $\Delta r \rightarrow 0$ for $m_{t} \sim 245 \mathrm{GeV}$.

We use the correlated average of the UA1 (Ref. 33) and UA2 (Ref. 34) values for $M_{W}$ and $M_{Z}$ given in Table V, along with the theoretical expressions in Table IV, obtained by expanding (48) and (50) to $O\left(s^{2}\right) \cdot \sin ^{2} \theta_{W}$ is determined by fitting to the data simultaneously with the mixing angles. 
TABLE IV. Experimental quantities and their theoretical expressions to lowest nontrivial order in $s^{2}$. $F\left(s^{2}\right)$ is defined by $F\left(s^{2}\right) \equiv\left(\hat{G}_{\mu} / G_{\mu}\right)^{1 / 2}=1+\frac{1}{4}\left[\left(s_{L}^{e}\right)^{2}+\left(s_{L}^{v}\right)^{2}+\left(s_{L}^{\mu}\right)^{2}+\left(s_{L}^{\mu}\right)^{2}+\left(s_{L}^{\nu}\right)^{2}\right]$. The quantities $x_{e}, x_{\mu}, z_{e}$, and $z_{\mu}$ are defined as $x_{e} \equiv\left(s_{R}^{e}\right)^{2}\left(s_{R}^{v}\right)^{2}$,

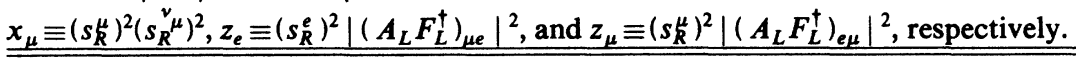

\begin{tabular}{|c|c|c|}
\hline Quantity & Expression & Source \\
\hline$M_{W}$ & $\frac{A_{0}}{\sin \theta_{W}(1-\Delta r)^{1 / 2}}\left[2-F\left(s^{2}\right)\right]$ & \\
\hline$M_{Z}$ & $\frac{M_{W}}{\cos \theta_{W}}$ & \\
\hline $1-\sum_{i=1}^{3}\left|V_{u l}\right|^{2}$ & $\left(s_{L}^{\mu}\right)^{2}-\left(s_{L}^{\mu}\right)^{2}-\left(s_{L}^{\nu} \mu\right)^{2}+\sum_{t=4}^{n}\left|V_{u t}\right|^{2}$ & Universality \\
\hline & $\begin{array}{l}+\left|V_{u d}\right|^{2}\left[\left(s_{L}^{d}\right)^{2}-2 \operatorname{Re}\left(\kappa_{u d}\right)\right] \\
+\left|V_{u s}\right|^{2}\left[\left(s_{L}^{s}\right)^{2}-2 \operatorname{Re}\left(\kappa_{u s}\right)\right]\end{array}$ & \\
\hline $1-\frac{R_{\pi}(\text { expt })}{R_{\pi}(\text { theory })}$ & $\left(s_{L}^{e}\right)^{2}+\left(s_{L}^{{ }^{e}}\right)^{2}-\left(s_{L}^{\mu}\right)^{2}-\left(s_{L}^{{ }^{\mu}}\right)^{2}$ & \\
\hline $1-\frac{R_{K}(\text { expt })}{R_{K}(\text { theory })}$ & $\left(s_{L}^{e}\right)^{2}+\left(s_{L}{ }^{e}\right)^{2}-\left(s_{L}^{\mu}\right)^{2}-\left(s_{L}{ }^{\mu}\right)^{2}$ & \\
\hline $1-\left(\frac{G_{\tau e}}{G_{\tau \mu}}\right)^{2}$ & $\left(s_{L}^{e}\right)^{2}+\left(s_{L}{ }^{v}\right)^{2}-\left(s_{L}^{\mu}\right)^{2}-\left(s_{L}{ }^{\mu}\right)^{2}$ & \\
\hline $1-\left(\frac{G_{\tau \mu}}{G_{\mu e}}\right)^{2}$ & $\left(s_{L}^{\tau}\right)^{2}+\left(s_{L}{ }^{\tau}\right)^{2}-\left(s_{L}^{e}\right)^{2}-\left(s_{L}{ }^{e}\right)^{2}$ & $\tau$ lifetime \\
\hline $1-\frac{4}{3} \rho$ & $x_{e}+x_{\mu}+z_{e}+z_{\mu}$ & Electron spectrum \\
\hline$\frac{1}{3}\left(1-\frac{4 \delta}{3}\right)$ & $x_{\mu}-x_{e}+z_{\mu}-z_{e}$ & Energy dependence of $e^{-}$asymmetry \\
\hline$\frac{1}{2}\left(1-\xi^{\prime}\right)$ & $x_{e}+z_{e}$ & $e^{-}$helicity \\
\hline$\frac{1}{2}\left(1-P_{\mu} \frac{\xi \delta}{\rho}\right)$ & $x_{\mu}$ & $e^{-}$asymmetry near end point \\
\hline$\frac{1}{2}\left(1-P_{\mu} \xi\right)$ & $2 x_{e}+2 z_{e}-z_{\mu}$ & $e^{-}$asymmetry \\
\hline$\frac{1}{2}\left(1-P_{e^{-}}\right)$ & $x_{e}$ & $e^{-}$helicity in $\beta$ decay \\
\hline $1-\frac{4}{3} \rho_{\tau \rightarrow \mu}$ & $\simeq\left(s_{R}^{\tau}\right)^{2}\left(s_{R}^{v}\right)^{2}+\left(s_{R}^{\tau}\right)^{2}\left|\left(A_{L} F_{L}^{\dagger}\right)_{\mu \tau}\right|^{2}$ & $\tau$ decay spectrum \\
\hline $1-\frac{4}{3} \rho_{\tau \rightarrow e}$ & $\simeq\left(s_{R}^{\tau}\right)^{2}\left(s_{R}^{v^{\tau}}\right)^{2}+\left(s_{R}^{\tau}\right)^{2}\left|\left(A_{L} F_{L}^{\dagger}\right)_{e \tau}\right|^{2}$ & \\
\hline $1-\sum_{i=1}^{3}\left|V_{c i}\right|^{2}$ & $\begin{array}{l}\simeq\left(\left|V_{c s}\right|^{2}+\left|V_{c d}\right|^{2}\right)\left(s_{L}^{c}\right)^{2} \\
+\left|V_{c s}\right|^{2}\left(s_{L}^{s}\right)^{2}+\sum_{i=4}^{n}\left|V_{c i}\right|^{2}\end{array}$ & Universality \\
\hline$\varepsilon_{L}(i)$ & $\left.F_{1}\left(s^{2}, \kappa\right)\left[t_{3}^{i}\left(c_{L}^{i}\right)^{2}-\sin ^{2} \theta_{W} q^{i}\right]\right]$ & $\begin{array}{l}\text { Deep-inelastic scattering; } \\
F_{1}\left(s^{2}, \kappa\right) \equiv \frac{1-\frac{\Lambda_{\mu}}{2}\left(s_{L}{ }^{\nu}{ }^{\mu}\right)^{2}}{}\end{array}$ \\
\hline & & 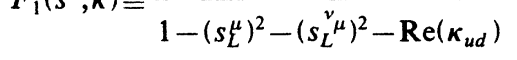 \\
\hline$g_{V}^{e}$ & $\left.F_{2}\left(s^{2}\right)\left[-\frac{1}{2}\left(c_{L}^{e}\right)^{2}-\frac{1}{2}\left(s_{R}^{e}\right)^{2}+2 \sin ^{2} \theta_{W}\right]\right]$ & Low-energy $v_{\mu} e:$ \\
\hline$g_{A}^{e}$ & $F_{2}\left(s^{2}\right)\left[-\frac{1}{2}\left(c_{L}^{e}\right)^{2}+\frac{1}{2}\left(s_{R}^{e}\right)^{2}\right]$ & $F_{2}\left(s^{2}\right) \equiv \frac{1-\frac{\Lambda_{\mu}}{2}\left(s_{L}{ }^{\nu}\right)^{2}}{1-\left(s_{L}^{\mu}\right)^{2}-\left(s_{L}^{v} \mu\right)^{2}}$ \\
\hline$g_{V}^{e}$ & $F_{1}\left(s^{2}, \kappa\right)\left[-\frac{1}{2}\left(c_{L}^{e}\right)^{2}-\frac{1}{2}\left(s_{R}^{e}\right)^{2}+2 \sin ^{2} \theta_{W}\right]$ & High-energy $v_{\mu} e$ \\
\hline$g_{A}^{e}$ & $F_{1}\left(s^{2}, \kappa\right)\left[-\frac{1}{2}\left(c_{L}^{e}\right)^{2}+\frac{1}{2}\left(s_{R}^{e}\right)^{2}\right]$ & \\
\hline$V^{\prime}$ & $F\left(s^{2}\right)\left\{t_{3}^{i}\left[\left(c_{L}^{i}\right)^{2}+\left(s_{R}^{i}\right)^{2}\right]-2 \sin ^{2} \theta_{W} q^{i}\right\}$ & Weak-electromagnetic interference \\
\hline $\boldsymbol{A}^{i}$ & $F\left(s^{2}\right)\left\{t_{3}^{i}\left[\left(c_{L}^{i}\right)^{2}-\left(s_{R}^{i}\right)^{2}\right]\right\}$ & \\
\hline
\end{tabular}


TABLE V. Experimental quantities. The stated errors include both experimental and theoretical uncertainties. The standardmodel (SM) expectations are for the global best fit value $\sin ^{2} \theta_{W}=0.23$.

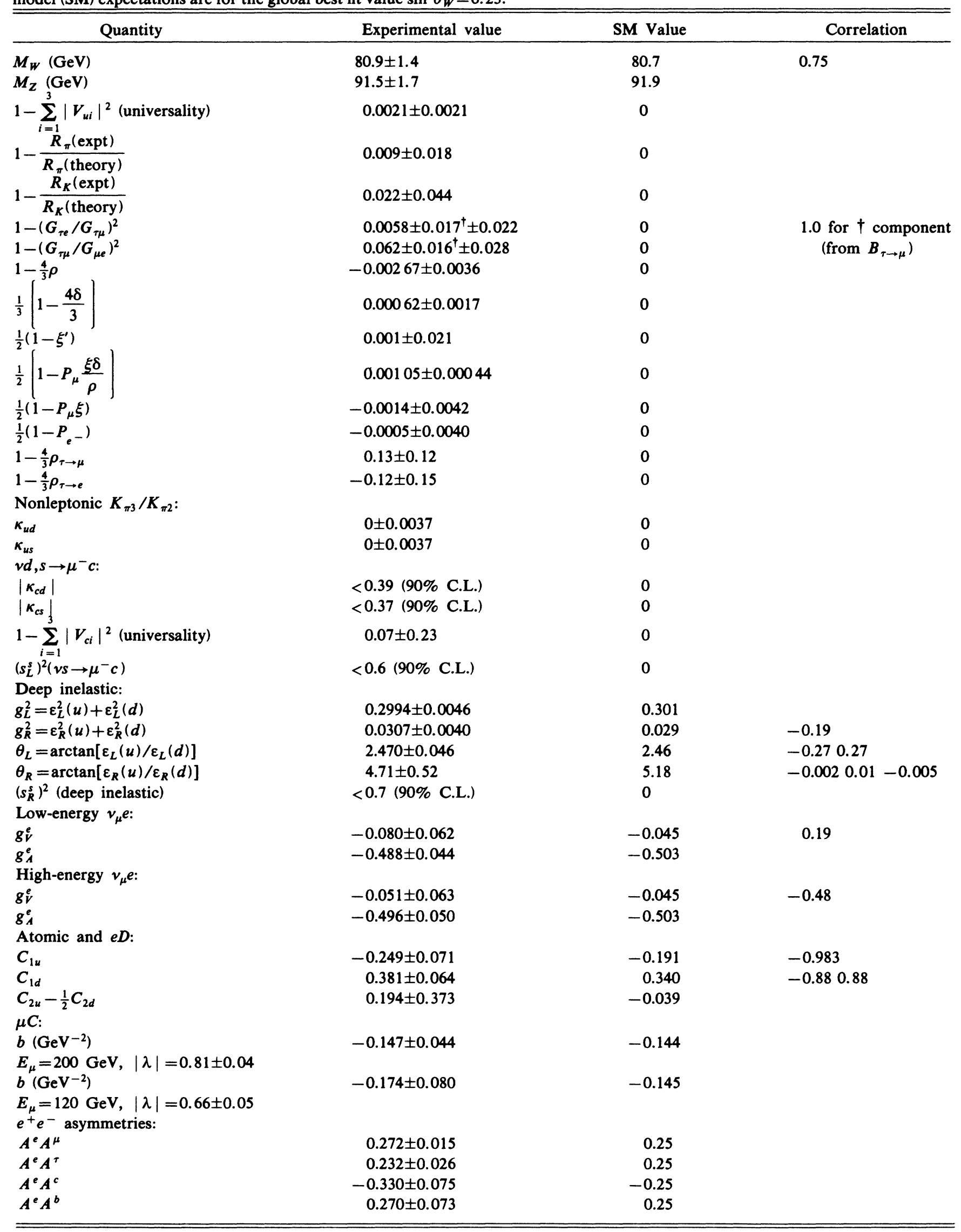




\section{B. Charged-current universality}

Universality tests constrain the relative strengths of weak amplitudes and, therefore, the relative sizes of lefthanded mixing angles.

The apparent KMC mixing angles $V_{u i}$ (we suppress the subscript $L$ ) $\operatorname{are}^{23}$

$$
\begin{aligned}
& \left|V_{u d}\right|=0.9744 \pm 0.0010 \\
& \left|V_{u s}\right|=0.220 \pm 0.002 \\
& 0.002<\left|V_{u b}\right|<0.012
\end{aligned}
$$

$\left|V_{u d}\right|$ is determined from superallowed $\beta$ decay and $\left|V_{u s}\right|$ from $K_{13}$ (mainly $K_{e 3}$ ) and the vector parts of hyperon decay. Hence, they depend only on hadronic vector currents, which are largely free of theoretical uncertainties. (We do not use constraints that depend on exclusive hadronic axial-vector matrix elements, because the hadronic couplings such as $g_{A}$ are not known independently to sufficient accuracy to disentangle the effects of exotic mixings.) The range for $\left|V_{u b}\right|$ [from $B \rightarrow p \bar{p} \pi$ 's and limits on $\Gamma(b \rightarrow u l v) / \Gamma(b \rightarrow c l v)]$ suffers from considerable theoretical uncertainty, but for all reasonable values $\left|V_{u b}\right|$ is too small to be of relevance for universality.

From (51),

$$
\left|V_{u d}\right|^{2}+\left|V_{u s}\right|^{2}+\left|V_{u b}\right|^{2}=0.9979 \pm 0.0021 \text {, }
$$

in excellent agreement with the prediction of unity for three-family universality.

$V_{u d}$ is extracted from the $\beta$-decay data by dividing the measured coefficient $G_{\mu} V_{u d}$ of the vector current by $G_{\mu}$ from $\mu$ decay. However, in the presence of mixing the actual coefficient of the (hadronic) vector part of the four-Fermi interaction is

$$
G_{\mu} V_{u d}=\hat{G}_{\mu} c_{L}^{e} c_{L}^{v_{e}}\left(\hat{V}_{L u d} c_{L}^{u} c_{L}^{d}+\hat{V}_{R u d} s_{R}^{u} s_{R}^{d}\right),
$$

where the second term is the induced RHC from (24). Using (49) one finds the relation

$$
V_{u d}=\frac{\hat{V}_{L u d} c_{L}^{u} c_{L}^{d}+\hat{V}_{R u d} s_{R}^{u} s_{R}^{d}}{c_{L}^{\mu} c_{L}^{v_{\mu}}} .
$$

Similarly, the apparent and true values of $V_{u s}$ are related by

$$
V_{u s}=\frac{\hat{V}_{L u s} c_{L}^{u} c_{L}^{s}+\hat{V}_{R u s} s_{R}^{u} s_{R}^{s}}{c_{L}^{\mu} c_{L}^{{ }^{\nu}}} .
$$

Combining (52), (54), (55), and the unitarity relation

$$
\sum_{i=1}^{n}\left|\widehat{V}_{L u i}\right|^{2}=1
$$

one obtains the unitarity constraint on $1-\sum_{i=1}^{3}\left|V_{u i}\right|^{2}$ given in Tables IV and $V$. In Table IV we have replaced $\widehat{V}_{L i j}$ with the measured $V_{i j}$ in the coefficients of $O\left(s^{2}\right)$ terms, allowed for possible mixings with extra sequential or vector doublets, ignored the negligible modifications to $V_{u b}$ and $V_{u i}, i \geq 4$, due to exotic mixing, and introduced the symbol

$$
\kappa_{i j}=s_{R}^{i} s_{R}^{j} \frac{\hat{V}_{R i j}}{\hat{V}_{L i j}} .
$$

The universality constraint is extremely stringent. Unlike the $W$ and $Z$ masses, however, the mixing terms do not all have the same sign so there is a possibility of cancellations. $s_{L}^{e}$ and $s_{L}^{v_{e}}$ do not enter because $\beta, K_{e 3}$, hyperon, and $\mu$ decay are all modified in the same way by $c_{L}^{e}$ and $c_{L}^{v_{e}}$.

\section{C. $e-\mu-\tau$ universality}

The ratio $R_{\pi} \equiv \Gamma(\pi \rightarrow e v) / \Gamma(\pi \rightarrow \mu v)$ has been measured very precisely. ${ }^{35,36}$ The experimental value relative to the standard-model theoretical prediction ${ }^{37}$ is

$$
\begin{aligned}
\frac{R_{\pi}(\text { expt })}{R_{\pi}(\text { theory })} & =0.991 \pm 0.018 \\
& =\frac{\left(c_{L}^{v^{e}}\right)^{2}\left(c_{L}^{e}\right)^{2}+\left(s_{R}^{v_{e}}\right)^{2}\left(s_{R}^{e}\right)^{2}}{\left(c_{L}{ }^{\mu}\right)^{2}\left(c_{L}^{\mu}\right)^{2}+\left(s_{R}^{{ }^{\mu}}\right)^{2}\left(s_{R}^{\mu}\right)^{2}}
\end{aligned}
$$

(the pion decay constant from the hadronic matrix element cancels in the ratio). The theoretical expression for $1-R_{\pi}(\operatorname{expt}) / R_{\pi}$ (theory), expanded to $O\left(s^{2}\right)$, and the experimental values are given in Tables IV and $V$, along with the (less precise) values of the analogous quantity $R_{K} \equiv \Gamma(K \rightarrow e v) / \Gamma(K \rightarrow \mu v)$.

The relative branching ratios $^{21}$ for $\tau \rightarrow e \nu \bar{v}$ and $\tau \rightarrow \mu \nu \bar{v}$ determine the ratio of effective Fermi couplings $G_{\tau e}$ and $G_{\tau \mu}$ and provide yet another constraint on $\mu$-e universality. Finally, the $\tau$ decay rate ${ }^{21}$

$$
\frac{\Gamma(\tau \rightarrow \mu \nu \bar{v})_{\text {expt }}}{\Gamma(\tau \rightarrow \mu \nu \bar{v})_{\text {theory }}}=0.938 \pm 0.016 \pm 0.028
$$

(the two errors are from the $\tau \rightarrow \mu$ branching ratio and total lifetime, respectively) tests $\tau$-e universality and constrains the effective Fermi coupling constant $G_{\tau \mu}$. The decay rate is about $2 \sigma$ below expectations (in the direction that could be accounted for by $\tau$ or $\nu_{\tau}$ mixings), but we do not consider the effect to be statistically compelling.

\section{Induced leptonic right-handed currents}

There are a number of very precise measurements in muon decay that are sensitive to induced RHC (Ref. 38). The relevant quantities are

$$
\begin{aligned}
& \left|g^{L L}\right|^{2}=\left(c_{L}^{e}\right)^{2}\left(c_{L}^{\mu}\right)^{2}\left(c_{L}^{v_{e}}\right)^{2}\left(c_{L}^{{ }{ }}\right)^{2}, \\
& \left|g^{R R}\right|^{2}=\left(s_{R}^{e}\right)^{2}\left(s_{R}^{\mu}\right)^{2}\left(s_{R}^{v_{e}}\right)^{2}\left(s_{R}^{v_{\mu}}\right)^{2}, \\
& \left|g^{L R}\right|^{2}=\left(c_{L}^{e}\right)^{2}\left(s_{R}^{\mu}\right)^{2}\left[\left(c_{L}^{v_{e}}\right)^{2}\left(s_{R}^{v_{\mu}}\right)^{2}+\left|\left(A_{L} F_{L}^{\dagger}\right)_{e \mu}\right|^{2}\right], \\
& \left|g^{R L}\right|^{2}=\left(s_{R}^{e}\right)^{2}\left(c_{L}^{\mu}\right)^{2}\left[\left(s_{R}^{v_{e}}\right)^{2}\left(c_{L}^{v_{\mu}}\right)^{2}+\left|\left(A_{L} F_{L}^{\dagger}\right)_{\mu e}\right|^{2}\right],
\end{aligned}
$$

where $g^{a b}$ is the coefficient of the four-Fermi interaction involving an $a$-handed electron and $b$-handed muon, and $\left(A_{L} F_{L}^{\dagger}\right)_{i j}$ is a possible $\Delta L= \pm 2\left(N_{O L}^{0}-N_{E L}^{0}\right)$-induced mixing between $v_{e L}$ and $v_{\mu L}^{c}$ or $v_{e L}^{c}$ and $v_{\mu L}$. (A general dis- 
cussion of muon decay involving neutrino mixing and other sources of lepton-number violation will be presented elsewhere. ${ }^{16}$ ) The observables relevant for RHC, involving polarizations, asymmetries, and the electron spectrum, are all of $O\left(s^{4}\right)$ and are listed in Tables IV and V.

The electron helicity from $\beta$ decay $^{39}$ and the spectrum parameter from leptonic $\tau$ decay $^{21}$ are also presented.

\section{E. Induced hadronic right-handed currents}

Donoghue and Holstein ${ }^{40}$ have argued that the standard-model PCAC (partial conservation of axialvector currents) predictions for nonleptonic $K_{\pi 3}$ amplitudes (relative to $K_{\pi 2}$ amplitudes) are successful at the $10 \%$ level, and that sets surprisingly stringent limits on hadronic RHC (the new operators associated with the RHC have matrix elements that are enhanced by the $\Delta I=\frac{1}{2}$ rule). Interpreting their limits on $\bar{u}_{R} d_{R}$ and $\bar{u}_{R} s_{R}$ currents as $1 \sigma$ errors, we obtain the limits on $\kappa_{u d}$ and $\kappa_{u s}$ [Eq. (57)] in Table V. $\operatorname{Re}\left(\kappa_{u d}\right)$ and $\operatorname{Re}\left(\kappa_{u s}\right)$ are also stringently constrained by universality (Tables IV and V).

These limits are much more stringent than those derivable from $\beta$ and hyperon decay, which depend on poorly known axial-vector matrix elements. For example, $g_{A}(0)$ is obtained by dividing the observed axial-vector coefficient

$$
G_{\mu} V_{u d} g_{A}(0) \equiv \hat{G}_{\mu} c_{L}^{e} c_{L}^{v_{e}}\left(\hat{V}_{L u d} c_{L}^{u} c_{L}^{d}-\hat{V}_{R u d} s_{R}^{u} s_{R}^{d}\right) \hat{g}_{A}(0)
$$

by $G_{\mu} V_{u d}$ from (53), so that $g_{A} / \widehat{g}_{A} \simeq 1-2 \kappa_{u d}$. If one assumes that the theoretical predictions of $\hat{g}_{A}$ (e.g., from the Adler-Weisberger or Goldberger-Treiman relations) are accurate to $5 \%$, then one has a limit of only $\simeq 0.025$ on $\left|\kappa_{u d}\right|$. Similarly, the CERN-Dortmond-HeidelbergSaclay (CDHS) group ${ }^{41}$ has used the $y$ distributions in deep inelastic $v N \rightarrow \mu^{-} X$ at large $x$ (at small $x$ it is difficult to disentangle RHC from the antiquark content of the nucleon) to set a limit on RHC. They obtain, in our notation, $\left|\kappa_{u d}\right|<0.095$ [90\% C.L. (confidence limit)].

The CDHS Collaboration ${ }^{41}$ has also measured $v$ - and $\bar{v}$-induced dimuon production, due to $v d, v s \rightarrow \mu^{-} c$ and $\bar{v} \bar{d}, \overline{v s} \rightarrow \mu^{+} \bar{c}$. From the measured $y$ distributions they have derived limits on induced $d \rightarrow c$ or $s \rightarrow c$ RHC, given in Table $\mathrm{V}$.

The lepton spectra in semileptonic $b$ and $c$ decays are in excellent agreement with the standard model, ${ }^{42}$ and could in principle constrain $\kappa_{c b}, \kappa_{c s}$, etc. We have not attempted to do so quantitatively, however, because of complications from bound state, QCD end point, and other effects.

\section{F. Limits on $s_{L}^{s}$ and $V_{c i}, i>3$}

The mixing of the $s_{L}$ quark is constrained by universality $\left[\left(s_{L}^{s}\right)^{2} \leq 0.10\right]$, but that limit can be evaded by cancellations. The best quantitative limit that cannot be finetuned away is from the semileptonic decay rate ${ }^{43}$
$\Gamma\left(D^{0} \rightarrow K^{-} e^{+} v_{e}\right)=(7.7 \pm 1.0 \pm 1.1) \times 10^{10} \quad \mathrm{~s}^{-1}$. For reasonable assumptions concerning the vector form factor $^{43}$ this implies $V_{c s}=0.94 \pm 0.12$, where

$$
V_{c s} \simeq c_{L}^{c} c_{L}^{s} \hat{V}_{L c s}+s_{R}^{c} s_{R}^{s} \hat{V}_{R c s} .
$$

Similarly, $\quad\left|V_{c d}\right| \simeq\left|c_{L}^{c} \hat{V}_{L c d}\right|=0.207 \pm 0.024$ from $^{41,23}$ $v d \rightarrow c X$. (In $V_{c s}$ and $V_{c d}$ it is justified to neglect the mixings of $\nu_{\mu}, \mu$, and the first-family fermions.) Combining this with the negligibly small ${ }^{23}\left|V_{c b}\right|=0.043 \pm 0.008$ and using the unitarity of $\hat{V}_{L}$, one obtains the constraint on $1-\sum_{i=1}^{3}\left|V_{c i}\right|^{2}$ in Tables IV and V. (In Table IV we have neglected $\kappa_{c s}$ because of the $v s \rightarrow \mu^{-} c y$ distributions $^{41}$ and neglected exotic mixings for $V_{c i}$, $i \geq 3$.) This leads to the very weak 90\%-C.L. limit $\left(s_{L}^{s}\right)^{2}<0.5$.

An independent limit is obtained from the measured rate $^{41}$ for $v s \rightarrow c \mu^{-}$, which is dominated by the lefthanded current (because of the $y$ distribution). In the three-family standard model (for which $V_{c s} \sim \cos \theta_{C}$ ) one obtains $^{41} \bar{S} / \bar{U}=0.46 \pm 0.08$, where $S \equiv \int_{0}^{1} x s(x) d x$ is the integrated moment of the $s$ quark in the nucleon. Allowing for exotic mixing and sequential doublets, this becomes

$$
\frac{\left(c_{L}^{c}\right)^{2}\left(c_{L}^{s}\right)^{2}\left|\widehat{V}_{L c s}\right|^{2}}{\cos ^{2} \theta_{C}} \frac{\bar{S}}{\bar{C}}=0.46 \pm 0.08 .
$$

Since $\left(c_{L}^{c}\right)^{2}$ and $\left|\hat{V}_{L c s}\right|^{2} \leq 1$, the reasonable assumption $\bar{S} / \bar{U} \leq 1$ implies a rather conservative lower bound of $\sim 0.4$ on $\left(c_{L}^{s}\right)^{2}$, so that $\left(s_{L}^{s}\right)^{2}<0.6$. Surprisingly, these and the even weaker limit on $\left(s_{R}^{s}\right)^{2}$ discussed below, are considerably less stringent than those on $c, b$, and $\tau$ mixing.

\section{G. Neutral-current constraints}

The charged-current constraints are quite stringent, but they only restrict the $s_{L}^{i}$ and the $\kappa_{i j}$. Also, they allow fine-tuned cancellations between different mixings. Further constraints are provided by the flavor-diagonal weak neutral current, including deep-inelastic neutrino scattering $(\nu N \rightarrow v X)$, elastic $v_{\mu} e \rightarrow v e$, atomic parity violation, polarization and mixed asymmetries in $e D \rightarrow e X$ and $\mu C \rightarrow \mu X$, respectively, and forward-backward asymmetries in $e^{+} e^{-} \rightarrow \mu^{+} \mu^{-}, \tau^{+} \tau^{-}, c \bar{c}$, and $b \bar{b}$. In particular, the neutral-current couplings of the $u, d, e$, and $\mu$ (both left and right handed) have been extracted from the data in a model-independent way, ${ }^{2}$ so that both $s_{L}^{i}$ and $s_{R}^{i}$ are constrained. Furthermore, the axial-vector couplings of the $\tau, c$, and $b$ have been determined, constraining $\left(s_{L}^{i}\right)^{2}+\left(s_{R}^{i}\right)^{2}$ for these particles. There is even a weak constraint on $\left(s_{R}^{s}\right)^{2}$.

\section{Deep-inelastic neutrino scattering}

The most precise measurements of the neutrino-quark interaction are from the ratios

$$
R_{v}=\frac{\sigma_{v N \rightarrow v X}}{\sigma_{v N \rightarrow \mu^{-} X}}, \quad R_{\bar{v}}=\frac{\sigma_{\bar{v} N \rightarrow \bar{v} X}}{\sigma_{\bar{v} N \rightarrow \mu^{+} X}},
$$

measured for (approximately) isoscalar targets and for proton targets. Exotic mixings affect not only the ha- 
dronic and leptonic neutral and charged currents [Eqs. (23), (24), (43), and (46)], but also the determination of subsidiary quantities such as the quark distribution functions, the charm threshold (important in the chargedcurrent denominator), and the KMC matrix elements (for the charged current) needed for the theoretical expressions for $R_{v}$ and $R_{\bar{v}}$. Fortunately, for isoscalar targets $R_{v}$ and $R_{\bar{v}}$ are insensitive to the details of the quark distributions: to first approximation they depend only on the ratio $\bar{Q} / Q$ of antiquarks to quarks in the nucleon, where $\bar{Q} \equiv \int_{0}^{1} x \bar{q}(x) d x$. It can be shown that, to an excellent approximation, the effects of exotic mixing cancel in the experiments which determine this ratio and the other ratios (e.g., $\bar{S} / \bar{U}$ and $D / U$ ) needed for refined treatments of $\boldsymbol{R}_{v, \bar{v}}$. Similarly, the charm threshold effects are essentially taken from experiment ${ }^{41}$ and are not affected.

However, the effects on the KMC elements are important. Fortunately, the charged-current cross sections are dominated by $u \leftrightarrow d$ transitions. It is an adequate approximation to replace the expression for charged-current cross sections by

$$
\begin{aligned}
\frac{1}{\sigma_{0}} \sigma\left(v_{\mu} N \rightarrow \mu^{-} X\right) & =\left(c_{L}^{{ }^{\mu}}\right)^{2}\left(c_{L}^{\mu}\right)^{2}\left(c_{L}^{\mu}\right)^{2}\left(c_{L}^{d}\right)^{2} \frac{\left|\hat{V}_{L u d}\right|^{2}}{\left|V_{u d}\right|^{2}} \\
& \simeq\left(c_{L}^{v^{\mu}}\right)^{4}\left(c_{L}^{\mu}\right)^{4}-2 \operatorname{Re}\left(\kappa_{u d}\right),
\end{aligned}
$$

where $\sigma_{0}$ is the canonical charged-current cross section computed in terms of the measured apparent KMC angles (we do not have to distinguish between $\hat{G}_{\mu}$ and $\boldsymbol{G}_{\mu}$ because these cancel in $\left.R_{v, \bar{v}}\right)$. The $c_{L}^{i}$ factors in the first expression are the direct effects of mixing, while the $\left|V_{L u d}\right|^{2} /\left|V_{u d}\right|^{2}$ factor is the indirect effect due to the $\mathrm{KMC}$ angle. In the second expression, obtained from Eq. (54), $\kappa_{u d}$ is not the direct effect of induced RHC in the cross section [these are of $O\left(\kappa^{2}\right)$ ], but rather enters through $\left|\widehat{V}_{\text {Lud }}\right| /\left|V_{\text {ud }}\right|$.

The neutral-current cross section is modified from the canonical form by the direct mixing effects in (23) and (46).

In the absence of mixing, the effective Lagrangian for neutrino-quark interactions is

$$
-\mathcal{L}^{\nu H}=\frac{4 G_{F}}{\sqrt{2}} \bar{v}_{L} \gamma^{\mu} v_{L} \sum_{a=L, R} \sum_{i=u, d, \ldots} \varepsilon_{a}(i) \bar{q}_{a}^{i} \gamma_{\mu} q_{a}^{i},
$$

where

$\varepsilon_{L}(i)=t_{3}^{i}-\sin ^{2} \theta_{W} q^{i}, \quad \varepsilon_{R}(i)=-\sin ^{2} \theta_{W} q^{i}$.

The experimental values ${ }^{44}$ of $g_{a}^{2} \equiv \varepsilon_{a}^{2}(u)+\varepsilon_{a}^{2}(d)$ and of $\theta_{a} \equiv \arctan \left[\varepsilon_{a}(u) / \varepsilon_{a}(d)\right], a=L$ or $R$, are shown in Table $\mathrm{V}$, along with the standard-model predictions for the global best-fit value $\sin ^{2} \theta_{W}=0.230$. [The values of the deep-inelastic and other neutral-current parameters in Table $\mathrm{V}$ incorporate small but non-negligible radiative corrections, as described in Ref. 2. We have not displayed these corrections in (67) or Table IV for simplicity, but include them in our numerical fits.]

The effects of exotic mixing (in both the neutral- and charged-current cross sections) can be incorporated by comparing the effective $\varepsilon_{L, R}(i)$ parameters in Table IV with the numerical constraints in Table V. It is apparent that the excellent agreement between the data and the standard-model prediction do not leave much room for exotic mixings.

Unlike the $c$ and $b$ quarks, there are no significant neutral-current constraints on the $s$ quark. As we have seen, charged-current universality implies reasonably small values for $\left(s_{L}^{s}\right)^{2}$ (unless there are fine-tuned cancellations). From the known s-quark fraction of the nucleon [Eq. (63)] one can set $^{45}$ the extremely weak limit $\left(s_{R}^{s}\right)^{2}<0.7$ (90\% C.L.) [i.e., complete mixing, $\left(c_{R}^{s}\right)^{2}=0$, is excluded]. Otherwise the $s_{R}$ neutral current would be significant and would lead to observable shifts in $R_{v}$ and especially $\boldsymbol{R}_{\bar{v}}$.

\section{Neutrino electron scattering}

The effective $v_{\mu} e$ interaction in the standard model is

$$
-\mathcal{L}^{v_{\mu} e}=\frac{2 G_{F}}{\sqrt{2}} \bar{v}_{L} \gamma^{\mu} v_{L} \bar{e} \gamma_{\mu}\left(g_{V}^{e}+g_{A}^{e}\right) e,
$$

where

$$
g_{V}^{e}=-\frac{1}{2}+2 \sin ^{2} \theta_{W}, \quad g_{A}^{e}=-\frac{1}{2},
$$

up to radiative corrections. Mixing effects modify $g_{V, A}^{e}$ according to (37) and (46).

A complication is that the $v_{\mu} e$ cross sections are measured relative to a charged-current reaction used for normalization and these cross sections are also affected by mixing. The low-energy BNL experiment ${ }^{46}$ E734 normalizes to quasielastic $v_{\mu} n \rightarrow \mu^{-} p$, while the higher-energy Fermilab and CERN experiments ${ }^{2}$ normalize mainly to deep-inelastic $v_{\mu} N \rightarrow \mu^{-} X$. The appropriate effective parameters which incorporate these normalization effects are given in Table IV, and separate low- and high-energy values for $g_{V, A}^{e}$ in Table $V$.

\section{Weak-electromagnetic interference}

A number of experiments measure weak-electromagnetic interference effects. These are only modified by the relation between $G_{\mu}$ and $\hat{G}_{\mu}$, and by direct neutralcurrent mixing effects [(23) and (46)], since electromagnetic amplitudes are not affected by mixing.

The atomic parity violation ${ }^{47}$ and polarized $e D$ asymmetry ${ }^{48}$ experiments measure the parity-violating couplings $C_{1 i}, C_{2 i}$ in Table $\mathrm{V}$, defined by

$$
-\mathcal{L}^{e H}=\frac{G_{F}}{\sqrt{2}} \sum_{i}\left(C_{1 i} \bar{e} \gamma_{\mu} \gamma^{5} e \bar{q}^{i} \gamma^{\mu} q^{i}+C_{2 i} \bar{e} \gamma_{\mu} e \bar{q}^{i} \gamma^{\mu} \gamma^{5} q^{i}\right)
$$

where in the standard model (up to radiative corrections)

$$
C_{1 i}=2 A^{e} V^{i}, C_{2 i}=2 V^{e} A^{i},
$$

where

$$
A^{i}=t_{3}^{i}, \quad V^{i}=t_{3}^{i}-2 \sin ^{2} \theta_{W} q^{i},
$$

are the axial-vector and vector neutral-current couplings of fermion $i$, respectively. Including mixing, (71) still 
holds provided $A^{i}$ and $V^{i}$ are modified as shown in Table IV.

The Bologna-CERN-Dubna-Munich-Saclay (BCDMS) Collaboration $^{49}$ measured the asymmetry between $\mu^{+}(-|\lambda|) C$ and $\mu^{-}(+|\lambda|) C$ deep-inelastic scattering where $\mp|\lambda|$ is the muon polarization. They measured a quantity $b$, proportional to the asymmetry, given by

$$
b=K\left(A^{\mu}-|\lambda| V^{\mu}\right)\left(A^{d}-2 A^{\mu}\right),
$$

where $K$ is a constant and the $A^{i}$ and $V^{i}$ are given in Table IV. The asymmetry is especially important for constraining $V^{\mu}$.

The forward-backward asymmetries ${ }^{2,8,50}$ in $e^{+} e^{-}$ $\rightarrow \mu^{+} \mu^{-}, \tau^{+} \tau^{-}, c \bar{c}$, and $b \bar{b}$ determine the product of axial-vector couplings $A^{e} A^{i}, \quad i=\mu, \tau, c, b$. Since $A^{i} \propto\left[1-\left(s_{L}^{i}\right)^{2}-\left(s_{R}^{i}\right)^{2}\right]$ the experimental results constrain both $\left(s_{L}^{i}\right)^{2}$ and $\left(s_{R}^{i}\right)^{2}$, i.e., because of the positivity of $s^{2}$ there is no possibility of cancellation.

\section{RESULTS}

There are enough constraints in Tables IV and $\mathrm{V}$ to simultaneously determine $\sin ^{2} \theta_{W}$, to constrain the mixings $\left(s_{L, R}^{i}\right)^{2}$ of all of the known $L$ and $R$ fermions, to limit the mixings $\left|V_{u i}\right|^{2}$ and $\left|V_{c i}\right|^{2}$ with sequential or vector doublets, and to limit all leptonic- and hadronic-induced right-handed charged currents except $b \leftrightarrow c, u$. In particular, (a) the universality constraints (and $M_{W, Z}$ ) restrict $\left(s_{L}^{i}\right)^{2}$ for $i=v_{e}, v_{\mu}, e, \mu, u$, and $d$, (b) the neutral-current limits $\left(s_{L}^{v_{\mu}}\right)^{2},\left(s_{L, R}^{i}\right)^{2}$ for $i=\mu, e, u$, and $d$, and the heavyfermion mixings, (c) $\mu$ and $\beta$ decay severely bound induced leptonic RHC, and (d) current-algebra results for nonleptonic $K_{\pi 3}$ decay and the $y$ distributions for $v d, v s \rightarrow \mu c$ constrain hadronic RHC. In this section we describe the results of (a) allowing only one parameter to vary, (b) allowing all parameters to vary simultaneously, and (c) specific models [which generally lead to limits between those of (a) and (b)].

We determine limits on mixings using a least-squares procedure for the data in Table V. One complication is the positivity constraint $\left(s_{L, R}^{i}\right)^{2} \geq 0$ on mixings. This actually leads to much stronger limits than would otherwise exist, since in many cases it precludes the possibility of cancellations. However, it also confuses the relation between the $\chi^{2}$ and the confidence level for a given set of parameters. We have therefore adopted the following procedure: (a) For each variable $x_{i}$ we determine a $\chi^{2}$ distribution using the data in Table $\mathrm{V}$. For each value of $x_{i}, \chi^{2}\left(x_{i}\right)$ is minimized with respect to all of the other parameters as they vary over their allowed ranges. (b) We assume a probability distribution

$$
P\left(x_{i}\right) d x_{i}=c_{i} e^{-x^{2}\left(x_{i}\right) / 2} d x_{i}
$$

for each $x_{i}$, with $c_{i}$ chosen so that $P\left(x_{i}\right)$ is properly normalized over the allowed range of $x_{i}$. (c) $90 \%$-C.L. upper limits on mixings are determined from $P\left(x_{i}\right)$.

Throughout we use $m_{t}=45 \mathrm{GeV}$ and $m_{H}=100 \mathrm{GeV}$ in the radiative corrections to neutral-current processes and to $M_{W, Z}$. Larger values of $m_{t}$ shift the central value of $\sin ^{2} \theta_{W}$ downwards ${ }^{2}$ but have no significant effects on the

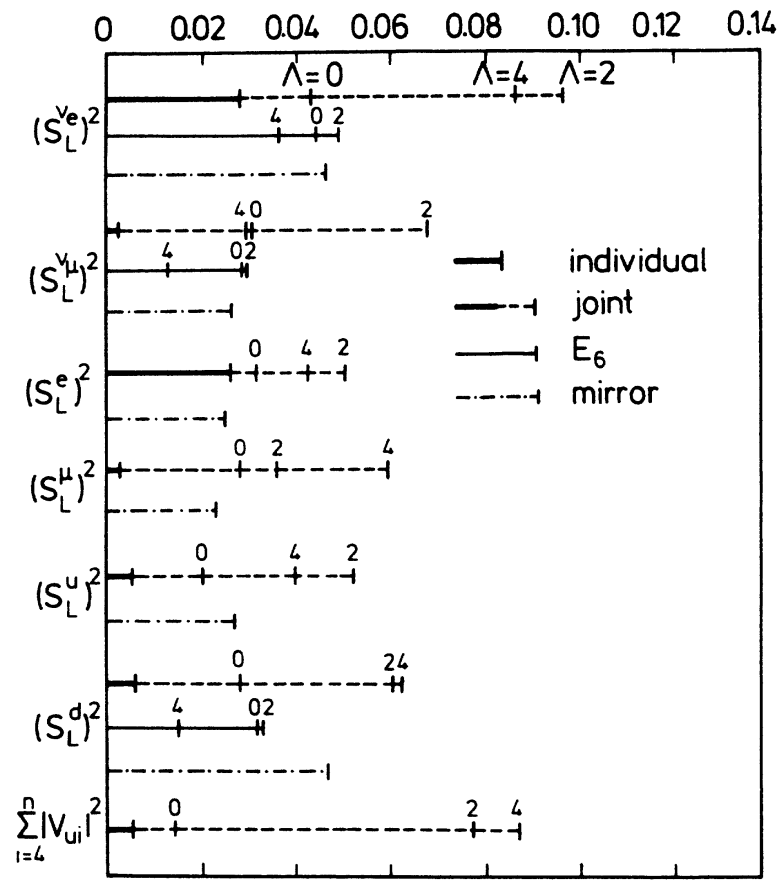

FIG. 1. 90\%-C.L. upper limits on mixings for the light lefthanded fermions, for individual fits (heavy solid lines), joint fits to all mixings (dashed lines), $\mathrm{E}_{6}$ models (solid lines), and the Hermitian mirror model (dotted-dashed line). The values of $\Lambda_{\mu}$ are indicated.

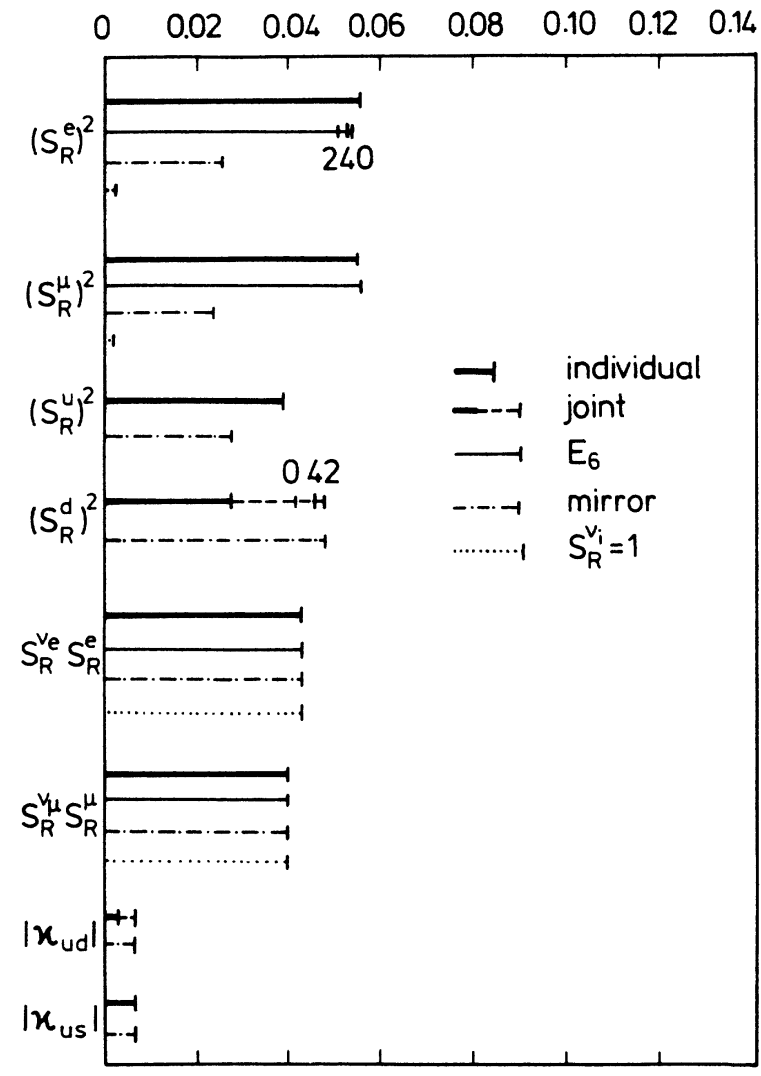

FIG. 2. 90\%-C.L. upper limits on mixings for light righthanded fermions. 
limits on exotic mixings. The $m_{H}$ dependence is negligible. The only neutrino neutral-current data that we use is for the $v_{\mu}$. For the parameter $\Lambda_{\mu}$ in (46), which ranges from 0 to 4 , we consider the representative special cases $\Lambda_{\mu}=0,2$, and 4 [see (47)].

The results are shown in Tables VI and VII and Figs. 1-3. The extremely stringent individual limits on $\left(s_{L}{ }^{{ }^{\mu}}\right)^{2}$, $\left(s_{L}^{\mu}\right)^{2},\left(s_{L}^{d}\right)^{2},\left(s_{L}^{u}\right)^{2}$, and on the mixing $\sum_{i=4}^{n}\left|V_{u i}\right|^{2}$ with extra $L$ doublets are from the universality relation $(52)$, while the much weaker limits on $\left(s_{L}^{v_{e}}\right)^{2}$ and $\left(s_{L}^{e}\right)^{2}$ are from $R_{\pi}(58)$.

In the joint fits the universality constraints can be partially evaded by cancellations. However, the combined effect of the neutral current and universality is to restrict
$\left(s_{L}^{v_{e}}\right)^{2},\left(s_{L}^{v_{\mu}}\right)^{2},\left(s_{L, R}^{i}\right)^{2}, i=e, \mu, u, d$, and $\sum_{i=4}^{n}\left|V_{u i}\right|^{2}$ to the few percent level.

The value $\sin ^{2} \theta_{W}=0.230 \pm 0.005$ obtained from the neutral-current data and $M_{W, Z}$ in the standard model ${ }^{2}$ is hardly affected by exotic mixings: one obtains $\sin ^{2} \theta_{W}=0.224 \pm 0.006,0.229 \pm 0.005$, and $0.224 \pm 0.005$, for $\Lambda_{\mu}=2,0$, and 4 , respectively. It is especially remarkable that the uncertainty in $\sin ^{2} \theta_{W}$ is not significantly increased, despite the large number of free parameters. This is a consequence of the number of precision experiments.

There is little correlation between the limits on $\left(s_{L}^{i}\right)^{2}$ and $\left(s_{R}^{i}\right)^{2}$, as is apparent from the fact that the $\left(s_{R}^{i}\right)^{2}$ limits are almost the same in the individual and joint fits.

TABLE VI. (a) 90\%-C.L. limits on the mixing angles for the cases in which each angle is fit separately, and in which all parameters are fit simultaneously. Absent entries imply that the best fit does not vary significantly with $\Lambda$. (b) Limits on the mixing angles for three particular models: $E_{6}$; mirror fermions with a Hermitian mass matrix; massless $R$-doublet neutrinos.

\begin{tabular}{|c|c|c|c|c|}
\hline & Individual & $\begin{array}{c}\text { (a) } \\
\Lambda_{\mu}=2 \\
\text { (singlets) }\end{array}$ & $\begin{array}{c}\text { Joint fit } \\
\Lambda_{\mu}=0 \\
\text { (sequential) }\end{array}$ & $\begin{array}{c}\Lambda_{\mu}=4 \\
(\Delta L= \pm 2)\end{array}$ \\
\hline$\left(s_{L}^{v e}\right)^{2}$ & 0.029 & 0.098 & 0.044 & 0.088 \\
\hline$\left(s_{L}^{v}{ }^{\nu}\right)^{2}$ & 0.0024 & 0.069 & 0.031 & 0.030 \\
\hline$\left(s_{L}^{e}\right)^{2}$ & 0.026 & 0.051 & 0.032 & 0.043 \\
\hline$\left(s_{L}^{\mu}\right)^{2}$ & 0.0021 & 0.036 & 0.028 & 0.060 \\
\hline$\left(s_{L}^{u}\right)^{2}$ & 0.0049 & 0.053 & 0.020 & 0.040 \\
\hline$\left(s_{L}^{d}\right)^{2}$ & 0.0054 & 0.061 & 0.028 & 0.063 \\
\hline$\sum_{i=4}^{n}\left|V_{u l}\right|^{2}$ & 0.005 & 0.078 & 0.014 & 0.088 \\
\hline$\left(s_{R}^{e}\right)^{2}$ & 0.055 & 0.052 & 0.054 & 0.050 \\
\hline$\left(s_{R}^{\mu}\right)^{2}$ & 0.054 & 0.055 & 0.052 & 0.051 \\
\hline$\left(s_{R}^{u}\right)^{2}$ & 0.038 & 0.034 & 0.035 & 0.036 \\
\hline$\left(s_{R}^{d}\right)^{2}$ & 0.027 & 0.047 & 0.041 & 0.045 \\
\hline$s_{R}^{v}{ }^{v} s_{R}^{e}$ & 0.042 & & 0.042 & \\
\hline${ }_{s_{R}{ }^{v}{ }^{\mu} s_{R}^{\mu}}^{\mu}$ & 0.039 & & 0.039 & \\
\hline$\left|\kappa_{u d}\right|$ & 0.0025 & & 0.006 & \\
\hline$\left|\kappa_{u s}\right|$ & 0.006 & & 0.006 & \\
\hline
\end{tabular}

(b)

\begin{tabular}{|c|c|c|c|c|c|}
\hline & $\Lambda_{\mu}=2$ & $\begin{array}{c}E_{6} \\
\Lambda_{\mu}=0\end{array}$ & $\Lambda_{\mu}=4$ & Mirror & $s_{R}^{v_{t}}=1$ \\
\hline$\left(s_{L}^{v e}\right)^{2}$ & 0.050 & 0.045 & 0.037 & 0.047 & \\
\hline$\left(s_{L}^{v}{ }^{v}\right)^{2}$ & 0.030 & 0.029 & 0.013 & 0.027 & \\
\hline$\left(s_{L}^{e}\right)^{2}$ & & & & 0.025 & \\
\hline$\left(s_{L}^{\mu}\right)^{2}$ & & & & 0.023 & \\
\hline$\left(s_{L}^{u}\right)^{2}$ & & & & 0.027 & \\
\hline$\left(s_{L}^{d}\right)^{2}$ & 0.033 & 0.032 & 0.015 & 0.047 & \\
\hline \multicolumn{6}{|c|}{$\sum_{i=4}^{n}\left|V_{u i}\right|^{2}$} \\
\hline $\begin{array}{l}t=4 \\
\left(s_{R}^{e}\right)^{2}\end{array}$ & 0.050 & 0.053 & 0.052 & 0.025 & 0.0018 \\
\hline$\left(s_{R}^{\mu}\right)^{2}$ & 0.055 & 0.055 & 0.054 & 0.023 & 0.0015 \\
\hline$\left(s_{R}^{u}\right)^{2}$ & & & & 0.027 & \\
\hline$\left(s_{R}^{d}\right)^{2}$ & & & & 0.047 & \\
\hline$s_{R}^{v e} s_{R}^{e}$ & & 0.042 & & 0.042 & 0.042 \\
\hline$s_{R}^{v}{ }^{\nu} s_{R}^{\mu}$ & & 0.039 & & 0.039 & 0.039 \\
\hline$\left|\kappa_{u d}\right|$ & & & & 0.006 & \\
\hline$\left|\kappa_{u s}\right|$ & & & & 0.006 & \\
\hline
\end{tabular}




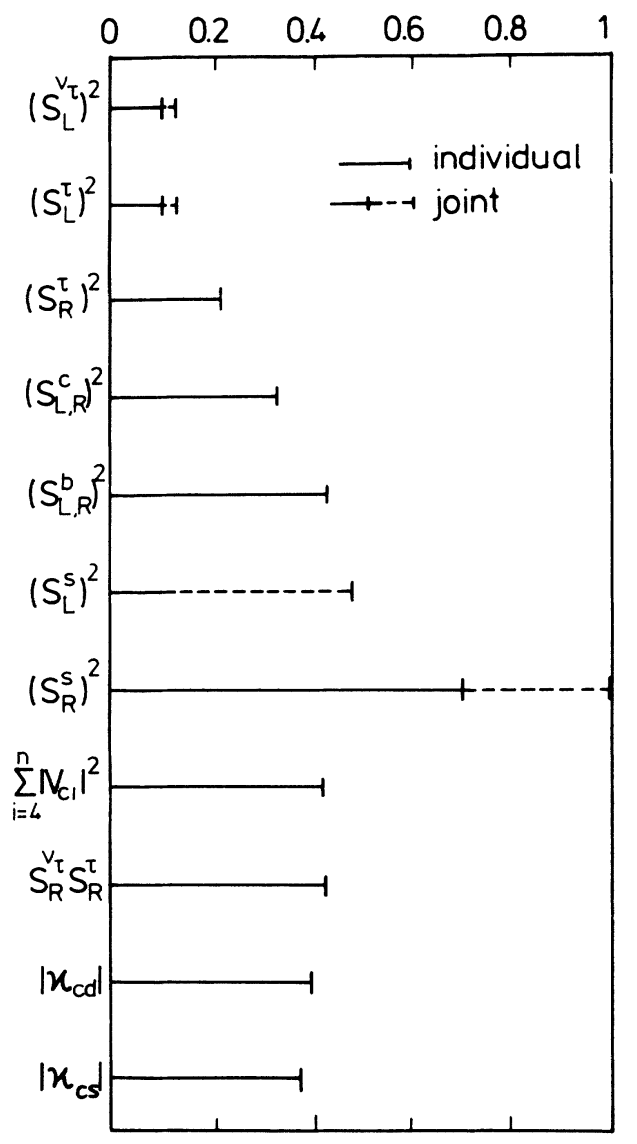

FIG. 3. 90\%-C.L. limits on mixings for the $s, b, c$, and $\tau$.

There is, however, a strong correlation between the $\left(s_{L}^{i}\right)^{2}$, especially for $i=v_{\mu}, \mu, u$, and $d$, because they must conspire to evade the universality constraint.

The limit on the hadronic RHC parameter $\left|\kappa_{u d}\right|$ is controlled by universality in the individual fit, and by $K_{\pi 3}$ in the joint fit. The limits on the leptonic RHC, $s_{R}^{e} s_{R}^{v_{e}}$ and $s_{R}^{\mu} s_{R}^{v_{\mu}}$, are mainly from $\mu$ decay. No individual limits on $s_{R}^{v_{e}}$ or $s_{R}^{v_{\mu}}$ can be derived because $s_{R}^{e}$ and $s_{R}^{\mu}$ are consistent with zero. For Dirac neutrinos $s_{R}^{v_{i}}$ describes the mixing of the light $\mathrm{SU}(2)$-singlet right-handed

TABLE VII. 90\%-C.L. limits on $v_{r}, \tau, s, c$, and $b$ mixings. The values are insensitive to $\Lambda$.

\begin{tabular}{lcc}
\hline & Individual & Joint \\
\hline$\left(s_{L}^{v^{\tau}}\right)^{2}$ & 0.10 & 0.13 \\
$\left(s_{L}^{\tau}\right)^{2}$ & 0.10 & 0.13 \\
$\left(s_{R}^{\tau}\right)^{2}$ & 0.22 & 0.22 \\
$\left(s_{L, R}^{c}\right)^{2}$ & 0.33 & 0.33 \\
$\left(s_{L, R}^{b}\right)^{2}$ & 0.43 & 0.43 \\
$\left(s_{L}^{s}\right)^{2}$ & 0.10 & 0.48 \\
$\left(s_{R}^{s}\right)^{2}$ & 0.7 & \\
$\sum_{i=4}^{n}\left|V_{c i}\right|^{2}$ & 0.42 & 0.42 \\
$s_{R}^{v} s_{R}^{\tau}$ & & \\
$\left|\kappa_{c d}\right|$ & 0.42 & 0.42 \\
$\left|\kappa_{c s}\right|$ & 0.39 & 0.39 \\
\hline & 0.37 & 0.37 \\
\hline
\end{tabular}

partners of the ordinary neutrinos into exotic $R$ doublets. For Majorana neutrinos, $s_{R}^{v_{i}}$ could also be generated by the $\Delta L= \pm 2$ mixing of the ordinary $v_{R}^{c}$ into $R$ doublets. (In the latter case one would also expect neutrinoless double-beta decay at some level. ${ }^{16}$ ) One could also have models in which there are neither light singlets nor $\Delta L= \pm 2$ mixings. Then $s_{R}^{v_{i}}=0$ automatically. Yet another possibility is discussed below.

Table VII and Fig. 3 contain the limits on the $s, c, b$, and $\tau$ mixings and induced RHC. These limits are much weaker than and largely decoupled from those in Table VI.

The results for three specific models are also shown in Table VI. These are the following. (a) The $\mathrm{E}_{6}$ model with all fermions assigned to 27-plets, as shown in Table II. No assumption is made concerning the Higgs structure, so all three kinds of neutrino mixing are possible. However, not all kinds of quark and charged-lepton exotics are present, so one obtains somewhat more stringent limits than in the general case. (b) Mirror fermions with a Hermitian mass matrix and Dirac neutrinos. (We consider $\Lambda=2$ only.) In this case the fermion mass matrices are diagonalized by the same unitary matrices for $L$ and $R$ so that $s_{L}^{i}=s_{R}^{i}$ for neutrinos, charged leptons, and quarks. As expected, the limits on the $s_{R}^{i}$ are somewhat better than for the general case in which $s_{R}^{i}$ and $s_{L}^{i}$ are unrelated. (c) A model in which there are exotic $R$ doublets involving massless neutrinos. This could come about in a mirror model in which the neutrinos are massless in both the ordinary and mirror sectors, for example. In this case we will use only the constraints from muon and $\beta$ decay on leptonic RHC with $s_{R}^{v_{e}, v_{\mu}}=1$; i.e., the $R$-doublet neutrinos are already massless, so no neutrino mixings are required. We do not use the other constraints because in many cases they were derived using the (no longer valid) assumption $s_{R}^{v_{i}} \ll 1$. In this case one obtains very stringent limits on $\left(s_{R}^{e}\right)^{2}$ and $\left(s_{R}^{\mu}\right)^{2}$.

From Tables VI and VII the limits on mixings are fairly stringent, at least for the $v_{e}, v_{\mu}, e, \mu, u$, and $d$. However, the corresponding constraints on the masses of the heavy neutrinos are rather weak. There is no rigorous model-independent relation between the mixings $s^{2}$ and the mass $\boldsymbol{M}$ of the heavy exotic fermions. However, two typical relations are

$$
\sin ^{2} \theta \sim\left(\frac{m}{M}\right)^{2} \quad \text { (quadratic seesaw) }
$$

and

$$
\sin ^{2} \theta \sim\left(\frac{m}{M}\right) \quad \text { (linear seesaw), }
$$

where $m$ and $M$ are the physical mass eigenvalues of the light and heavy fermions, respectively. (A large class of models will fall in the general range bounded by these two relations.)

The quadratic seesaw comes about, for example, if the fermion mass term (for one light and one heavy particle), is of the form 
TABLE VIII. Relation between the mixings $s_{L, R}^{2}$ and the heavy-mass eigenvalue $M$ (in $\mathrm{GeV}$ ) for quadratic $\left(s^{2} \sim m^{2} / M^{2}\right)$ and linear $\left(s^{2} \sim m / M\right)$ seesaw models. The light-fermion eigenvalue $m$ is taken to be $10 \mathrm{MeV}, 100 \mathrm{MeV}$, and $1 \mathrm{GeV}$, which is appropriate for the first, second, and third families, respectively.

\begin{tabular}{lcccccc}
\hline & \multicolumn{2}{c}{$M=m / s$} & \multicolumn{3}{c}{$M=m / s^{2}$} & (linear seesaw) \\
$s^{2}$ & $m=10 \mathrm{MeV}$ & $100 \mathrm{MeV}$ & $1 \mathrm{GeV}$ & $m=10 \mathrm{MeV}$ & $100 \mathrm{MeV}$ & $1 \mathrm{GeV}$ \\
\hline 0.001 & 0.32 & 3.2 & 32 & 10 & 100 & 1000 \\
0.002 & 0.22 & 2.2 & 22 & 5 & 50 & 500 \\
0.005 & 0.14 & 1.4 & 14 & 2 & 20 & 200 \\
0.01 & 0.10 & 1.0 & 10 & 1 & 10 & 100 \\
0.02 & & 0.71 & 7.1 & 0.5 & 5 & 50 \\
0.05 & 0.45 & 4.5 & 0.2 & 2 & 20 \\
0.1 & 0.32 & 3.2 & 0.1 & 1 & 10 \\
0.2 & 0.22 & 2.2 & & 0.5 & 5 \\
0.5 & 0.14 & 1.4 & & 0.2 & 2 \\
\hline \hline
\end{tabular}

$$
\left(\begin{array}{cc}
m & \epsilon \\
\epsilon & M
\end{array}\right),
$$

where $m$ is generated by the ordinary Higgs mechanism and $\epsilon \simeq m$ is a mixing mass of the same order of magnitude. Then the mass eigenvalues are $\sim m$ and $M$, and the mixing is $s_{L, R}^{2} \sim \epsilon^{2} / M^{2} \sim m^{2} / M^{2}$. The linear seesaw formula follows for a mass matrix of the form

$$
\left[\begin{array}{cc}
0 & \epsilon \\
\epsilon & M
\end{array}\right],
$$

i.e., if for some reason there is no direct mass term for the light fermions. Then the lighter eigenvalue $m \sim \epsilon^{2} / M$ is induced by mixing, and $s_{L, R}^{2} \sim \epsilon^{2} / M^{2} \sim m / M$. In Table VIII are shown the heavy-mass eigenvalues corresponding to various mixing angles for the two cases. For the light eigenvalues we use $m=10 \mathrm{MeV}, 100 \mathrm{MeV}$, and 1 $\mathrm{GeV}$, which are typical values appropriate to the first, second, and third families, respectively. Comparing Tables VI-VIII, the mass range probed by exotic mixings is very small-even compared to the direct limits in (3) - if the quadratic seesaw is correct. For the linear seesaw, on the other hand, there is sensitivity up to $\sim 50$ $\mathrm{GeV}$.

\section{DISCUSSION}

Many extensions of the standard model predict the existence of new fermions, often with exotic $\mathrm{SU}(2) \times \mathrm{U}(1)$ assignments. If one assumes there are no exotic electric charges or colors, then the only possibilities for the quarks and charged leptons are ordinary ( $L$-doublet, $R$ singlet) and exotic ( $L$-singlet, $R$-doublet) fermions, though some of the"ordinary" particles may be associated with new sequential families or new vector doublets or singlets. For the left-handed neutrinos (and their righthanded $\boldsymbol{C P}$ conjugates), there are three possibilities [Eq. (4)], all of which can mix if there are Majorana mass terms.

We have considered the indirect manifestations of exotic heavy fermions via their mixings with ordinary fermions. The most stringent limits are on the induced FCNC's between light particles (Table III). These are very strong but can be evaded for special forms of the mixing transformations. We have been mainly concerned with these special directions in parameter space. We showed that the absence of FCNC's between the light quarks and charged leptons allows an enormous simplification: it implies that each ordinary left- and right-handed particle can only mix with one unique heavy particle or linear combination of heavy particles-i.e., there is only one mixing angle per light degree of freedom. There is no evidence for the absence of FCNC between the neutrinos. However, it turns out that because one must sum over the unobserved flavors of the final neutrinos in weak processes one can again parametrize mixing effects by one effective mixing angle per neutrino, plus a bounded auxiliary parameter that describes the $S U(2) \times U(1)$ representation of the state with which the $v$ mixes. Exotic neutrino mixings can also generate lepton-flavor or total lepton-number violation. These will be discussed elsewhere. ${ }^{16}$

There are enough constraints from $M_{W, Z}$, chargedcurrent universality, limits on right-handed currents, and flavor-diagonal neutral currents to limit the possible mixings of all of the known fermions, even when all are allowed to mix simultaneously. The constraints are shown in Tables IV and V, and the 90\%-C.L. limits are shown in Tables VI and VII and Figs. 1-3. The limits on the mixings of the $v_{\mu L}, \mu_{L}^{-}, u_{L}$, and $d_{L}$ with exotic (or heavy doublet) fermions are very stringent $\left(s^{2}<0.002-0.005\right)$ if only one particle is allowed to mix at a time, and weaker by an order of magnitude if all of the particles are allowed to mix simultaneously. Limits on the mixings of the $v_{e L}, e_{L, R}^{-}, \mu_{R}^{-}, u_{R}$, and $d_{R}$ are in the range 0.02-0.06, while those for the $v_{\tau}, \tau, s, c$, and $b$ are much weaker (the weakest limits are for the $s$ quark). Slightly stronger limits are found in specific models. Limits are also given on possible induced hadronic and leptonic RHC. We have considered a very general class of models with exotic fermions. There remains the logical possibility that mixing effects could be compensated by other types of new physics, such as additional gauge bosons. However, given the large number of constraints in Tables IV and V, it seems highly unlikely that such effects could significantly alter the results.

The corresponding constraints on the masses of the 
heavy exotic fermions, shown in Table VIII, are rather weak: depending on the relation between the mixing angles and heavy particle mass the range of sensitivity varies from $\sim 50 \mathrm{GeV}$ to values below the direct production limits in (3). One can of course find models in which the heavy masses fall outside the range in Table VIII by a moderate factor. However, it is clear that despite the large number of high-precision weak-interaction experiments there is still considerable room for fairly light exotic fermions.

There have been many studies ${ }^{4,12}$ of the phenomenological implications of heavy fermions. However, a systematic analysis of the present and possible future direct detection limits, as well as of indirect limits from $\Gamma_{Z}$ and $\Gamma_{W} / \Gamma_{Z}$, for all of the possibilities in Table $I$, would be useful.

We have presented limits on all hadronic RHC transitions except $b \rightarrow c, u$. It should be possible to derive quantitative limits on these (and on $c \rightarrow s$ ) from the lepton spectra in semileptonic decays, which agree very well with standard-model predictions, but we have not attempted to do so.

One anticipated improvement is in the $Z$ mass. If $M_{Z}$ is measured to $\pm 20 \mathrm{MeV}$ at CERN LEP and the Stanford Linear Collider (SLC), and $\sin ^{2} \theta_{W}$ to \pm 0.0004 (from polarization asymmetries), then (assuming that $m_{t}$ has been directly determined) the major uncertainty is the Higgsboson mass dependence in $\Delta r( \pm 0.006)$. This would imply a sensitivity of order 0.01 for $\left(s_{L}^{v_{e}}\right)^{2}+\left(s_{L}^{e}\right)^{2}+\left(s_{L}^{v_{\mu}}\right)^{2}$ $+\left(s_{L}^{\mu}\right)^{2}$. This would still be less stringent than the present sensitivity from universality, but would not allow any fine-tuned cancellations.

\section{ACKNOWLEDGMENTS}

We are grateful to Istvan Montvay for helping stimulate our interest in this subject. This work was supported by DESY, by the Alexander von Humboldt-Stiftung (P.L.), and by Department of Energy Grant No. DEAC02-76-ERO-3071.

\section{APPENDIX}

(In this appendix the subscript $a=L$ or $R$ is suppressed.)

\section{Proof of Theorem 1 [Eq. (21)]}

Since $A$ is an $n \times n$ matrix, it can be written as

$$
A=\left(\begin{array}{lll}
\mathbf{y}_{1} & \cdots & \mathbf{y}_{n}
\end{array}\right), \quad A^{\dagger}=\left(\begin{array}{c}
\mathbf{y}_{1}^{\dagger} \\
\vdots \\
\mathbf{y}_{n}^{\dagger}
\end{array}\right),
$$

where $\mathbf{y}_{i}=\left(\left(y_{i}\right)_{1} \quad \cdots \quad\left(y_{i}\right)_{n}\right)^{T}$ is an $n$-component complex vector [i.e., $\left(\mathrm{y}_{i}\right)_{j} \equiv A_{j i}$ ]. But $A^{\dagger} A=c^{2}$ implies

$$
\left(A^{\dagger} A\right)_{i j}=\mathbf{y}_{i}^{\dagger} \cdot \mathbf{y}_{j}=\delta_{i j}\left(c^{i}\right)^{2} \text {. }
$$

Let us first assume that all of the $\left(c^{i}\right)^{2}$ are nonzero. Then one can define $\mathbf{x}_{i} \equiv \mathbf{y}_{i} / c^{i}, i=1, \ldots, n$, so that

$$
\mathbf{x}_{i}^{\dagger} \cdot \mathbf{x}_{j}=\delta_{i j}
$$

The $\mathbf{x}_{i}$ form a set of $n$ orthonormal vectors in an $n$ dimensional complex vector space. Hence, they are complete, i.e.,

$$
\sum_{k}\left(\mathbf{x}_{k}\right)_{i}\left(\mathbf{x}_{k}^{\dagger}\right)_{j}=\delta_{i j}
$$

One then has $A=\hat{A} c$, where $\hat{A}=\left(\begin{array}{lll}\mathbf{x}_{1} & \cdots & \mathbf{x}_{\eta}\end{array}\right)$. The unitarity of $\hat{A}$ follows from orthonormality $\left[\hat{A}^{\dagger} \hat{A}=I, \mathrm{Eq}\right.$. (A3)] and completeness $\left[\hat{A} \hat{A}^{\dagger}=I, \mathrm{Eq}\right.$. (A4)]. If one or more of the $c^{i}$ vanish, one can (i) define $\mathbf{x}_{i} \equiv \mathbf{y}_{i} / c^{i}$ for the nonzero $c^{i}$ and (ii) construct the remaining $\mathbf{x}_{i}$ (corresponding to the vanishing $c^{i}$ ) using the Gram-Schmidt procedure. The rest of the proof follows as above.

\section{Proof of Theorem 2 [Eq. (22)]}

$F$ is an $(m \times n)$-dimensional matrix, which can be written as

$$
F=\left(\begin{array}{lll}
\mathbf{w}_{1} & \cdots & \mathbf{w}_{n}
\end{array}\right), \quad F^{\dagger}=\left(\begin{array}{c}
\mathbf{w}_{1}^{\dagger} \\
\vdots \\
\mathbf{w}_{n}^{\dagger}
\end{array}\right),
$$

where the $\mathbf{w}_{i}$ are complex $m$ component vectors. From $F^{\dagger} F=s^{2}$ one has

$$
\left(F^{\dagger} F\right)_{i j}=\mathbf{w}_{i}^{\dagger} \cdot \mathbf{w}_{j}=\delta_{i j}\left(s^{i}\right)^{2} .
$$

For $\boldsymbol{m}=\boldsymbol{n}$ one can define a complete orthonormal set of vectors $\mathrm{z}_{i}=\mathrm{w}_{i} / s^{i}$ (if any of the $s^{i}$ should vanish the corresponding $\mathbf{z}_{i}$ can be constructed via the GramSchmidt procedure). Then $F=\hat{F}$, with $\hat{F}=\left(\begin{array}{lll}\mathbf{z}_{1} & \cdots & \mathbf{z}_{n}\end{array}\right)$ unitary, in analogy with Theorem 1.

For $m<n$ it follows from (A6) that at least $n-m$ of the $\mathbf{w}_{i}$ (and corresponding $s^{i}$ ) must vanish, because there can be at most $m$ independent vectors in an $m$ dimensional space. The interpretation is that the absence of FCNC's requires that each ordinary state mix with a unique exotic state. If there are $m<n$ exotic states, then only $m$ of the ordinary states can mix. One can define $\mathbf{z}_{i}=\mathbf{w}_{i} / s^{i}$ for the nonzero vectors, so that $F=\hat{F} s$, where

$$
\hat{F}=\left(\begin{array}{llllll}
\mathbf{z}_{1} & \cdots & \mathbf{z}_{m} & \mathbf{0} & \cdots & \mathbf{0}
\end{array}\right)
$$

(we have assumed $\mathbf{w}_{m+1} \cdots \mathbf{w}_{n}=0$ for notational convenience). Then it is easy to see that $\hat{F}^{\dagger} \hat{F}$ is the projection operator onto the $m$-dimensional subspace (of the $n$ ordinary states) which have nonzero light-heavy mixing, while $\hat{F} \hat{F}^{\dagger}=I$.

For $m>n$ one can again write $F=\hat{F} s$, where $\hat{F}=\left(\begin{array}{lll}\mathbf{z}_{1} & \cdots & \mathrm{z}_{n}\end{array}\right), \mathrm{z}_{i}=\mathbf{w}_{i} / s^{i}$. In this case $\hat{F} \hat{F}^{\dagger} \hat{F}=I$, while $\hat{F} \hat{F}^{\dagger}$ is the projection operator onto the $n$-dimensional subspace (of the $m$ exotic states) which actually participate in the mixing.

\section{Proof of Theorem 3}

From (10) and Theorems 1 and 2, one has

$$
U=\left(\begin{array}{ll}
\hat{A} c & E \\
\hat{F} s & G
\end{array}\right),
$$


where $\hat{A}$ is unitary. However, since the $n$ ordinary states all have the same $\mathrm{SU}(2) \times \mathrm{U}(1)$ transformation properties (and similarly for the $m$ exotic states), one can rewrite the theory in terms of a new weak basis

$$
\psi^{0^{\prime}}=\left(\begin{array}{c}
\psi_{O}^{0^{\prime}} \\
\psi_{E}^{0^{\prime}}
\end{array}\right)=\left(\begin{array}{cc}
B_{O} & 0 \\
0 & B_{E}
\end{array}\right)\left(\begin{array}{l}
\psi_{O}^{0} \\
\psi_{E}^{0}
\end{array}\right),
$$

where $B_{O}$ and $B_{E}$ are unitary $n \times n$ and $m \times m$ matrices. In the new basis one has

$$
U_{a}^{\prime}=\left(\begin{array}{cc}
B_{o} & 0 \\
0 & B_{E}
\end{array}\right) U_{a} .
$$

Choosing $B_{O}=\hat{A}^{\dagger}$ one obtains $\hat{A}^{\prime}=I$. Similarly, for $m=n$ one can choose $B_{E}=\hat{F}^{\dagger}$ so that $\hat{F}^{\prime}=I$. It is easy to show (using the forms for $\hat{F}$ displayed above) that for $m<n$ one can choose $B_{E}$ so that $\hat{F}^{\prime}$ is the identity when restricted to the $m$-dimensional subspace of ordinary states which mix, and that for $m>n, \hat{F}^{\prime}$ is the identity when restricted to the $n$-dimensional subspace of exotic states which mix.

\section{Interpretation}

From the unitarity relation (11) and Theorems 1-3, it is straightforward to display the necessary and sufficient condition for the absence of FCNC's. For the $n=m$ case the general unitary matrix $U$ reduces to the form

$$
U=\left(\begin{array}{cc}
\hat{A} & 0 \\
0 & \hat{F}
\end{array}\right]\left[\begin{array}{cc}
c & -s \\
s & c
\end{array}\right]\left[\begin{array}{ll}
I & 0 \\
0 & \hat{E}
\end{array}\right),
$$

where $\hat{E}$ is unitary. The first factor involves intergenerational mixing within the ordinary and exotic sectors. The middle term implies that light-heavy mixing is restricted to pairs of ordinary and exotic states. In the weak basis for which $\hat{A}=\hat{F}=I$, for example, the ordinary and exotic states $\psi_{i O}^{0}$ and $\psi_{i E}^{0}$ are just a rotation

$$
\begin{aligned}
& \psi_{i O}^{0}=\cos \theta^{i} \psi_{i l}-\sin \theta^{i}\left(\hat{E} \psi_{h}\right)_{i}, \\
& \psi_{i E}^{0}=\sin \theta^{i} \psi_{i l}+\cos \theta^{i}\left(\hat{E} \psi_{h}\right)_{i},
\end{aligned}
$$

of light-mass eigenstate $\psi_{i l}$ and a combination $\hat{E}_{i j} \psi_{j h}$ of heavy-mass eigenstates. A similar interpretation applies to the $n \neq m$ case, provided one restricts attention to the appropriate subspaces of light or heavy states which participate in the mixing.

\section{Proof of the neutral-current cross-section formula (46) to $O\left(s^{2}\right)$}

We will give the proof for the case that all massless neutrinos coincide with ordinary doublets $n_{O L}^{0}$ in the absence of mixing [so that the components of $F$ and $H$ are all of $O(s)$ ]. The proof is easily generalized to allow massless exotic doublets $\left(n_{E L}^{0}\right)$ and singlets $\left(n_{S L}^{0}\right)$.

The matrix $A$ can then be written as

$$
A=\left(\begin{array}{c}
A_{l} \\
A_{h}
\end{array}\right)
$$

where $A_{l}$ is a square $(p \times p)$-dimensional matrix relating the $p$ massless neutrinos to the first $p$ ordinary doublets and $A_{h}$ is $[(n-p) \times p]$-dimensional, describing the small $[O(s)]$ mixing of the $p$ massless $v$ 's into the $n-p$ heavy sequential or vector doublets. Of course, one can have $n-p=0$. One has

$$
A^{\dagger} A=A_{l}^{\dagger} A_{l}+A_{h}^{\dagger} A_{h}
$$

and

$$
\left(A A^{\dagger}\right)_{a a}=\left(A_{l} A_{l}^{\dagger}\right)_{a a}=c_{L}^{v_{a}^{2}},
$$

for $a \in 1, \ldots, p$.

In the absence of mixing, $A_{l}$ is unitary and one can choose a basis in the space of massless eigenstates for which $A_{l}=I$. Including mixing, one has [to $O\left(s^{2}\right)$ ]

$$
A_{l}=I-\frac{\Sigma^{2}}{2}
$$

where $\Sigma^{2}$ is a Hermitian matrix (by an appropriate choice of basis) with components of $O\left(s^{2}\right)$ [by (36)]. Hence, to $O\left(s^{2}\right)$,

$$
A_{l} A_{l}^{\dagger}=A_{l}^{\dagger} A_{l}=I-\Sigma^{2}
$$

so that $\left(\Sigma^{2}\right)_{a a}=\left(s_{L}^{v_{a}}\right)^{2}$.

Define the matrix $\Delta$ by

$$
I+\Delta=A^{\dagger} A-F^{\dagger} F \text {, }
$$

so that, from (36),

$$
\Delta=-2 F^{\dagger} F-H^{\dagger} H \text {. }
$$

Each component of $\Delta$ is, therefore, of $O\left(s^{2}\right)$. From (41), (45), (A12), and (A15),

$$
\frac{1}{\sigma_{0}} \sum_{i} \sigma\left(n_{a L} \rightarrow n_{i L}\right)=\frac{\left[A(I+2 \Delta) A^{\dagger}\right]_{a a}}{\left(A A^{\dagger}\right)_{a a}}=1+2 \Delta_{a a}
$$

to $O\left(s^{2}\right)$, where $a \in 1, \ldots, p$ (the range for which the theorem applies). The special cases in (47) follow immediately from (A18) and (A19) and the unitarity formula (36). More generally, from (36), (A13), (A14), and the positivity of $\left(F^{\dagger} F\right)_{a a}$, etc., one has

$$
\begin{aligned}
& \left(F^{\dagger} F\right)_{a a} \equiv \lambda_{F}^{a}\left(s_{L}^{v_{a}}\right)^{2}, \\
& \left(H^{\dagger} H\right)_{a a} \equiv \lambda_{H}^{a}\left(s_{L}^{v_{a}}\right)^{2},
\end{aligned}
$$

and

$$
\left(A_{h}^{\dagger} A_{h}\right)_{a a} \equiv \lambda_{A}^{a}\left(s_{L}^{v_{a}}\right)^{2}
$$

where $0 \leq \lambda \leq 1$ and $\lambda_{A}^{a}+\lambda_{F}^{a}+\lambda_{H}^{a}=1$. The theorem then follows from (A18) and (A19), with

$$
\Lambda_{a}=4 \lambda_{F}^{a}+2 \lambda_{H}^{a} .
$$


*Permanent address: Department of Physics, University of Pennsylvania, Philadelphia, PA 19104.

1J. Maalampi, K. Mursula, and M. Roos, Nucl. Phys. B207, 233 (1982); J. Maalampi and K. Mursula, Z. Phys. C 16, 83 (1982); Nucl. Phys. B269, 109 (1986); K. Enqvist, K. Mursula, and M. Roos, ibid. B226, 121 (1981); K. Enqvist, J. Maalampi, and M. Roos, Phys. Lett. B 176, 396 (1986); T. Rizzo, Phys. Rev. D 34, 2076 (1986); 34, 2163 (1986); P. M. Fishbane, R. E. Norton, and M. J. Rivard, ibid. 33, 2632 (1986); V. Barger, R. J. Phillips, and K. Whisnant, Phys. Rev. Lett. 57, 48 (1986); M. Gronau, C. N. Leung, and J. L. Rosner, Phys. Rev. D 29, 2539 (1984).

${ }^{2}$ For recent reviews of neutral-current experiments, see $U$. Amaldi et al., Phys. Rev. D 36, 2191 (1987); G. Costa et al., Nucl. Phys. B297, 244 (1988).

${ }^{3}$ S. Weinberg, Phys. Rev. Lett. 19, 1264 (1967); A. Salam, in Elementary Particle Theory: Relativistic Groups and Analyticity (Nobel Symposium No. 8), edited by N. Svartholm (Almqvist and Wiksell, Stockholm, 1969), p. 367; S. L. Glashow, J. Iliopoulos, and L. Maiani, Phys. Rev. D 2, 1285 (1970).

${ }^{4}$ For some representative references, see H. Baer, V. Barger, and R. J. N. Phillips, Phys. Rev. D 32, 688 (1985); W. J. Marciano and A. Sirlin, Phys. Rev. Lett. 56, 22 (1986); W. S. Hou, R. S. Willey, and A. Soni, ibid. 58, 1608 (1987); C. Albajar et al., Phys. Lett. B 185, 241 (1987); 197, 565(E) (1987); V. Barger and R. J. N. Phillips, ibid. 189, 473 (1987); R. M. Barnett and H. E. Haber, Phys. Rev. D 36, 2042 (1987); F. del Aguila et al., Phys. Lett. B 196, 531 (1987).

${ }^{5}$ Early references to mirror models can be found in the papers of Ref. 1. Some recent references are F. del Aguila, Ann. Phys. (N.Y.) 165, 237 (1985); T. Banks and M. Karliner, Nucl. Phys. B281, 399 (1987); S. Singh and N. K. Sharma, Phys. Rev. D 36, 160 (1987).

${ }^{6}$ See, for example, F. Wilczek and A. Zee, Phys. Rev. D 25, 553 (1982); J. Bagger et al., Nucl. Phys. B244, 247 (1984).

${ }^{7}$ For a general discussion, see I. Montvay, Phys. Lett. B 205, 315 (1988).

${ }^{8} \mathrm{~S}$. L. Wu, in Lepton and Photon Interactions, proceedings of the International Symposium on Lepton and Photon Interactions at High Energies, Hamburg, West Germany, 1987, edited by R. Rueckl and W. Bartel [Nucl. Phys. B Proc. Suppl. 3, 39 (1987)].

${ }^{9}$ UA1 Collaboration, C. Albajar et al., Phys. Lett. B 185, 241 (1987); 197, 565(E) (1987); S. Geer, in Proceedings of HEP '87, International Europhysics Conference on High Energy Physics, Uppsala, Sweden, 1987, edited by O. Botner (Uppsala University, Uppsala, Sweden, 1987).

${ }^{10}$ UA2 Collaboration, R. Ansari et al., Phys. Lett. B 195, 613 (1987).

${ }^{11}$ C. Albajar et al., Phys. Lett. B 198, 271 (1987), and references therein.

${ }^{12}$ See, for example, J. Rosner, Comments Nucl. Part. Phys. 15, 195 (1986); V. Barger et al., Phys. Rev. D 33, 1912 (1986); G. Couture and J. N. Ng, ibid. 35, 70 (1987); R. Rajpoot, ibid. 36, 1479 (1987); S. Godfrey, Phys. Lett. B 195, 78 (1987); J. L. Hewitt, ibid. 196, 223 (1987).

${ }^{13}$ Since we allow for Majorana neutrinos, the possible doublets

$$
\left(\begin{array}{c}
E^{+} \\
N
\end{array}\right)_{L, R}
$$

are not independent, but are related by $C P$ to the doublets

$$
\left(\begin{array}{c}
N \\
E^{-}
\end{array}\right]_{R, L} .
$$

${ }^{14}$ For a recent review, see P. Langacker, in Neutrino Physics, edited by H. V. Klapdor (Springer, Berlin, in press).

${ }^{15}$ B. W. Lee et al., Phys. Rev. Lett. 38, 937 (1977); B. W. Lee and R. E. Shrock, Phys. Rev. D 16, 1444 (1977); D. Wyler and L. Wolfenstein, Nucl. Phys. B218, 205 (1983); S. L. Glashow, Phys. Lett. B 187, 367 (1987); J. Bernabeu et al., ibid. 187, 303 (1987); J. W. F. Valle, ibid. 199, 432 (1987).

${ }^{16}$ P. Langacker and D. London, following paper, Phys. Rev. D 38, 907 (1988).

${ }^{17}$ Glashow, Iliopoulos, and Maiani (Ref. 3).

${ }^{18}$ We have checked that the contributions of virtual heavy particles to quantities such as the $K_{L}-K_{S}$ mass difference (e.g., in the box diagram involving $W$ or off-diagonal $Z$ exchange) are sufficiently small (at most comparable to ordinary diagrams and usually much smaller) for reasonable assumptions concerning the light-heavy mixing.

${ }^{19}$ B. A. Campbell et al., Int. J. Mod. Phys. A2, 831 (1987).

${ }^{20} \mathrm{R}$. Eichler, in Lepton and Photon Interactions (Ref. 8), p. 389.

${ }^{21}$ H. J. Gerber, in Proceedings of HEP '87 (Ref. 9); B. Barish and R. Stroynowski, Phys. Rep. 157, 1 (1988).

${ }^{22}$ B. Gittelman and S. Stone, Cornell Report No. CLNS 87/81 (unpublished).

${ }^{23} \mathrm{~A}$. Sirlin, in Lepton and Photon Interactions (Ref. 8), p. 417.

${ }^{24}$ For $n>m, \widehat{V}_{R}$ is unitary when restricted to the subspaces of $\psi_{l R}^{u}$ and $\psi_{l R}^{d}$ which mix with heavy quarks (Appendix). For $n<m, \widehat{V}_{R}$ is nonunitary in general, because the $\psi_{l R}^{u}$ and $\psi_{l R}^{d}$ each mix into (generally different) $n$-dimensional subspaces of the $\psi_{E R}^{0}$. For example, for one ordinary family but several exotic $R$ doublets, $u_{R}$ and $d_{R}$ could mix into different doublets, so the $\widehat{V}_{R u d}$ could vanish or be less than unity.

${ }^{25}$ For example, a single Dirac neutrino has (in conventional notation) the four components $\nu_{L} \leftrightarrow v_{R}^{c}$ (doublets) and $N_{R} \leftrightarrow N_{L}^{c}$ (singlets), where $v_{L}$ and $N_{R}$ are leptons and $v_{R}^{c}, N_{L}^{c}$ are antileptons. In our notation we would denote $\left(v_{L}, N_{L}^{c}\right)$ $\rightarrow\left(n_{1 L}, n_{2 L}\right)$ and $\left(v_{R}^{c}, N_{R}\right) \rightarrow\left(n_{1 R}^{c}, n_{2 R}^{c}\right)$.

${ }^{26}$ Because the Majorana mass matrix is symmetric one always has $U_{L} K=U_{R}^{*}$, where $K$ is a matrix of phases (Ref. 14) (unless there are degeneracies). For our present case of massless $v$ 's and unobserved heavy $v$ 's one can choose $K=I$.

${ }^{27}$ In writing (37) - (39) we have not exploited the degeneracy of the light-mass-eigenstate neutrinos (i.e., they are assumed to be effectively massless). Since there is nothing other than the weak currents to distinguish the different light states, one can rewrite them as $n_{l L}=D_{L} n_{l L}^{\prime}$ and $n_{l R}^{c}=D_{R} n_{l R}^{c \prime}$, where $D_{L}$ and $D_{R}$ are unitary. It is not, in general, possible to use this freedom to diagonalize $A_{L}^{v}$ or $F_{R}^{v}$, however.

${ }^{28}$ This is strictly only possible for $m_{R}^{e}=n_{R}^{e}$. For $m_{R}^{e}<n_{R}^{e}$, (38) still applies as long as $n-m$ of the $s_{R}^{e}$ vanish. For $m_{R}^{e}>n_{R}^{e}$, (38) holds if $F_{R}^{v}$ is restricted to the subspace of $R$ doublets which mix. Neither case leads to any practical difficulties.

${ }^{29}$ For $\mu$ decay and, in principle, leptonic $\tau$ decay, the effect is more complicated because of the possibility of interference between the two neutrinos. This will be discussed more fully in a separate paper.

${ }^{30}$ If the wrong helicity neutrino $n_{a R}^{c}$ produced in (40) is mainly an SU(2) singlet, $n_{S R}^{0^{c}}$, as expected for Dirac neutrinos, then the cross section for the $n_{a R}^{c}$ to rescatter is $\sigma\left(n_{a R}^{c} \rightarrow e_{a R}\right) / \sigma_{0}=\left(s_{R}^{e}\right)^{2}\left(s_{R}{ }^{a}\right)^{2}$. If it is mainly an ordinary doublet $n_{O R}^{0^{c}}$ as could occur for Majorana neutrinos, the rescattering cross section is unsuppressed. However, for charged-current rescattering an antilepton (e.g., $e_{R}^{+}$) is produced rather than a lepton.

${ }^{31}$ If the $n_{i L}$ have small masses the components $n_{i L}$ evolve with different phases, leading to the normal neutrino oscillation 
phenomena. Since neutrino oscillations have not been established and, in fact, are largely excluded at levels relevant to the reactions considered here, we ignore them.

${ }^{32}$ A. Sirlin, Phys. Rev. D 22, 971 (1980); 29, 89 (1984); see also Ref. 2.

${ }^{33}$ UA1 Collaboration, G. Arnison et al., Phys. Lett. 166B, 484 (1986).

${ }^{34}$ UA2 Collaboration, R. Ansari et al., Phys. Lett. B 186, 440 (1987).

${ }^{35}$ Particle Data Group, M. Aguilar-Benitez et al., Phys. Lett. 170B, 1 (1986).

${ }^{36}$ The most precise single experiment is D. A. Bryman et al., Phys. Rev. Lett. 50, 7 (1983), which obtains $0.983 \pm 0.011$ relative to expectation. However, we use the world average from Ref. 35.

${ }^{37}$ T. Goldman and W. Wilson, Phys. Rev. D 15, 709 (1977).

${ }^{38}(\rho)$ S. E. Derenzo et al., Phys. Rev. 181, $1854(1969) ;\left(P_{\mu} \xi \delta / \rho\right)$ A. Jodidio et al., Phys. Rev. D 34, 1967 (1986); 37, 237(E) (1988); ( $\delta$ ) B. Balke et al., ibid. 37, 587 (1988); ( $\left.\xi^{\prime}\right)$ H. Burkard et al., Phys. Lett. 150B, 242 (1985); $\left(P_{\mu} \xi\right)$ I. Beltrami et al., Phys. Lett. B 194, 326 (1987). For reviews, see Refs. 21 and 35.

${ }^{39}$ E. D. Commins and P. H. Bucksbaum, Weak Interactions of Leptons and Quarks (Cambridge University Press, Cambridge, England, 1983); F. W. J. Koks and J. Van Klinken, Nucl. Phys. A272, 61 (1976).

${ }^{40}$ J. F. Donoghue and B. R. Holstein, Phys. Lett. 113B, 382 (1982).
${ }^{41}$ CDHS Collaboration, H. Abramowicz et al., Z. Phys. C 12, 225 (1982) $\left(v d \rightarrow \mu^{-} u\right) ; 15,19$ (1982) $\left(v d, v s \rightarrow \mu^{-} c\right)$.

${ }^{42}$ B. Gittelman and S. Stone, Cornell Report No. CLNS 87/81 (unpublished); G. Altarelli et al., Nucl. Phys. B208, 365 (1982); N. Cabibbo, G. Corbo, and L. Maiani, ibid. B155, 93 (1979); B. Grinstein, N. Isgur, and M. Wise, Phys. Rev. Lett. 56, 298 (1986); L. Angelini et al., Phys. Lett. B 172, 447 (1986).

${ }^{43}$ E691 Collaboration, K. Sliwa, Proceedings of the XXIII Rencontre de Moriond (unpublished).

${ }^{44}$ Amaldi et al. (Ref. 2). The numbers in Table $\mathrm{V}$ differ slightly from those in Ref. 2 because they are determined from deepinelastic scattering. The corresponding values in Ref. 2 also include $v_{\mu} p \rightarrow v p$ elastic scattering, which we do not use here because of its nontrivial dependence on $\hat{g}_{A} / g_{A}$, and coherent pion production.

${ }^{45}$ P. Langacker (unpublished).

${ }^{46}$ E734 Collaboration, L. A. Ahrens et al., Phys. Rev. Lett. 51, 1514 (1983); 54, 18 (1985).

${ }^{47}$ For reviews, see M. A. Bouchiat and L. Pottier, Science 234, 1203 (1986); C. A. Piketty, in Weak and Electromagnetic Interactions in Nuclei, edited by H. V. Klapdor (Springer, Berlin, 1986), p. 603.

${ }^{48}$ C. Y. Prescott et al., Phys. Lett. 84B, 524 (1979).

${ }^{49}$ A. Argento et al., Phys. Lett. 120B, 245 (1983).

${ }^{50}$ R. Marshall, Rutherford Report No. RAL-87-031 (unpublished). 